\title{
Longitudinal variations in the gastrointestinal microbiome of the white shrimp, Litopenaeus vannamei
}

Estefanía Garibay-Valdez Equal first author, 1 Francesco Cicala Equal first author, 2 , Reyes $^{3}$, Francisco Vargas-Albores ${ }^{1}$, Teresa Gollas-Galván ${ }^{1}$, Luis Rafael Martínez-Córdova ${ }^{4}$, Kadiya Calderón ${ }^{4}$

${ }^{1}$ Tecnología de Alimentos de Origen Animal, Centro de Investigación en Alimentación y Desarrollo, Hermosillo, Sonora, México

2 Innovación Biomédica, Centro de Investigación Científica y de Educación Superior de Ensenada, Ensenada, Baja California, México

3 Universidad Autónoma de Baja California, Ensenada, Baja California, Mexico

4 Departamento de Investigaciones Científicas y Tecnológicas de la Universidad de Sonora, Universidad de Sonora, Hermosillo, Sonora, Mexico

Corresponding Author: Marcel Martinez-Porchas

Email address: marcel@ciad.mx

The shrimp gut is a long digestive structure that includes the Foregut (stomach), Midgut (hepatopancreas), and Hindgut (intestine). Each component has different structural, immunity, and digestion roles. Given these three gut digestive tract components' significance, we examined the bacterial compositions of the Foregut, Hindgut, and Midgut digestive fractions. Those bacterial communities' structures were evaluated by sequencing the V3 hypervariable region of the 16S rRNA gene, while the functions were predicted by PICRUSt2 bioinformatics workflow. Also, to avoid contamination with environmental bacteria, shrimp were maintained under strictly controlled conditions. The pairwise differential abundance analysis revealed differences among digestive tract fractions. The families Rhodobacteraceae and Rubritalaceae registered higher abundances in the Foregut fraction, while in the Midgut, the families with a higher proportion were Aeromonadaceae, Beijerinckiaceae, and Propionibacteriaceae. Finally, the Cellulomonadaceae family resulted in a higher proportion in the Hindgut. Regarding the predicted functions, amino acid and carbohydrate metabolism pathways were the primary functions registered for Foregut microbiota; conversely, pathways associated with the metabolism of lipid, terpenoids, and polyketides, were detected in the Midgut fraction. In the Hindgut, pathways like the metabolism of cofactors and vitamins along with energy metabolism were enriched. Structural changes were followed by significant alterations in functional capabilities, suggesting that each fraction's bacteria communities may carry out specific metabolic functions. Results indicate that white shrimp's gut microbiota is widely related to the fraction analyzed across the digestive tract. Overall, our results suggest a role for the dominant bacteria in each digestive tract fraction, contributing with a novel insight into the bacterial community. 
1 Longitudinal variations in the gastrointestinal

2 microbiome of the white shrimp, Litopenaeus

3 vannamei

4

5

6

Estefanía Garibay-Valdez ${ }^{*}$, Francesco Cicala ${ }^{2 *}$, Marcel Martínez-Porchas ${ }^{1}$, Ricardo GómezReyes $^{3}$, Francisco Vargas-Albores ${ }^{1}$, Teresa Gollas-Galván ${ }^{1}$, Luis Rafael Martínez-Córdova ${ }^{4}$, Kadiya Calderón ${ }^{4}$

${ }^{1}$ Centro de Investigación en Alimentación y Desarrollo, A. C., Carretera Gustavo Enrique Astiazarán Rosas, N0. 46, Hermosillo, Sonora, México. C.P. 83304. Tel: +52(662) 2892400.

${ }^{2}$ Departamento de Innovación Biomédica, Centro de Investigación Científica y de Educación Superior de Ensenada, Ensenada, Baja California, México. CP 22860. Tel. +52 (646)175-0500 .

${ }^{3}$ Universidad Autónoma de Baja California, Carretera Transpeninsular Ensenada-Tijuana NO. 3917, Ensenada, Baja California. C.P. 22860. Tel: +52 (646) 1750710

${ }^{4}$ Departamento de Investigaciones Científicas y Tecnológicas de la Universidad de Sonora. Blvd Luis Donaldo Colosio entre Reforma y Sahuaripa, Hermosillo, Sonora. C.P. 83000. Tel: +52 (662) 259.2169.

Corresponding Author:

Marcel Martínez-Porchas ${ }^{1}$

Carretera Gustavo Enrique Astiazarán Rosas, N0. 46, Hermosillo, Sonora, México Email address: marcel@ciad.mx

* Equal first authors

\section{Abstract}

The shrimp gut is a long digestive structure that includes the Foregut (stomach), Midgut

(hepatopancreas), and Hindgut (intestine). Each component has different structural, immunity, and digestion roles. Given these three gut digestive tract components' significance, we examined the bacterial compositions of the Foregut, Hindgut, and Midgut digestive fractions. Those bacterial communities' structures were evaluated by sequencing the V3 hypervariable region of the 16S rRNA gene, while the functions were predicted by PICRUSt2 bioinformatics workflow. Also, to avoid contamination with environmental bacteria, shrimp were maintained under strictly controlled conditions. The pairwise differential abundance analysis revealed differences among 
35 digestive tract fractions. The families Rhodobacteraceae and Rubritalaceae registered higher

36 abundances in the Foregut fraction, while in the Midgut, the families with a higher proportion

37 were Aeromonadaceae, Beijerinckiaceae, and Propionibacteriaceae. Finally, the

38 Cellulomonadaceae family resulted in a higher proportion in the Hindgut. Regarding the

39 predicted functions, amino acid and carbohydrate metabolism pathways were the primary

40 functions registered for Foregut microbiota; conversely, pathways associated with the

41 metabolism of lipid, terpenoids, and polyketides, were detected in the Midgut fraction. In the

42 Hindgut, pathways like the metabolism of cofactors and vitamins along with energy metabolism

43 were enriched. Structural changes were followed by significant alterations in functional

44 capabilities, suggesting that each fraction's bacteria communities may carry out specific

45 metabolic functions. Results indicate that white shrimp's gut microbiota is widely related to the

46 fraction analyzed across the digestive tract. Overall, our results suggest a role for the dominant

47 bacteria in each digestive tract fraction, contributing with a novel insight into the bacterial

48 community.

50 Introduction

51 The white shrimp, Litopenaeus vannamei, is the penaeid shrimp species with a high economic

52 value worldwide. Despite the great production of this species, there are many problems to solve to

53 boost the shrimp aquaculture industry. In particular, shrimp diseases lead to significant economic

54 losses. The dysbiosis of the intestine can contribute to several shrimp diseases, such as acute

55 hepatopancreatic necrosis disease (AHPND) and white feces syndrome (WFS) (Hossain et al.

56 2021; Huang et al. 2020a). Recently, Wu et al. (2021) revealed that dysbiosis in the

57 hepatopancreatic microbiota of the crayfish (Procambarus clarkii) is associated with disease

58 outbreaks. 
59 The white shrimp interacts with a wide variety of microbes colonizing the internal and external

60 body (Das et al. 2008). The microbial communities thriving in the digestive tract can play crucial

61 roles in the animal's digestion, nutrition, and immune response (Sommer \& Bäckhed 2013). For

62 example, the digestive tract microbiota provides a considerable enzymatic capability, leading to

63 manage most aspects of host physiology. Also, some tissue-specific pathways regulate the

64 homeostatic relationship with microbiota (Belkaid \& Hand 2014).

65

66

67

In shrimp, the gut microbiota is implicated in health and disease (Zheng et al. 2017), optimal growth, and culture stages (Garibay-Valdez et al. 2020). Gut microbiota provides the host with beneficial functions. For instance, the microbiotas main roles include nutrient absorption, digestive enzyme production, generation of essential elements for the host's metabolism and immune response by activating mechanisms of protection against pathogens, and the competitive exclusion of pathogenic bacteria (Gómez \& Balcázar 2008; Rowland et al. 2018; Tzuc et al. 2014). Besides, dysbiosis in the host intestinal microbiota is associated with shrimp diseases as white feces syndrome (WFS) rather than a single pathogen (Huang et al. 2020b).

Although previous studies assessed the bacterial composition in the shrimp intestine, few studies address the structural and functional changes of the microbiota among the different shrimp's digestive tract fractions. However, recent studies have emphasized the diversity and role of the digestive tract microbiota in terrestrial (insects and mammals) and aquatic animals (fish) according to the digestive tract fraction (Brune \& Dietrich 2015; Jandhyala et al. 2015). The digestive tract fraction supports different microbial communities that are driven by distinct metabolic processes (Nielsen et al. 2017). The shrimp gut is a long digestive structure that includes the Foregut (stomach), Midgut (hepatopancreas), and Hindgut (intestine), being the intestine, the most studied fraction (Cornejo-Granados et al. 2018; Gao et al. 2019; Li et al. 2019). Each component has 
82 different structural and digestive roles, including absorption, digestion, and excretion. Also, the 83 hepatopancreas (Midgut gland) is a vital organ responsible for digestion, absorption, and storage 84 of nutrients in crustaceans, and it also plays a crucial role in regulating host innate immunity 85 (Cornejo-Granados et al. 2017; Cheung et al. 2015; Gao et al. 2019; Rőszer 2014). Understanding

86

87 88

the entire shrimp digestive tract microbiota leads to a better comprehension of host-microbiota interactions and their regulatory mechanisms linked to the examined fraction from the digestive tract.

The Midgut or hepatopancreas is also a habitat of numerous bacteria that could provide specific shrimp development and physiological functions. There are only a few studies about the hepatopancreatic microbiota. For instance, Cornejo-Granados et al. (2017) described the differences in bacterial diversity in shrimp intestine and hepatopancreas; results revealed Vibrio shilonii, Faecalibacterium prausnitzii, and Aeromonas taiwanensis in the intestine. However, Pantoea agglomerans and Photobacterium angustum were detected in the hepatopancreas, and the differences were associated with the digestive tract fraction (hepatopancreas or intestine) and the environment type (wild or cultured). Cheung et al. (2015) evaluated the bacterial communities from the intestine (Midgut and Hindgut), Foregut (stomach), and hepatopancreas of the cherry shrimp Neocaridina denticulata. Results revealed distinct bacterial composition in the hepatopancreas concerning the gut during the gonadal development. The mycobiota in the shrimp digestive tract was evaluated, registering a high diversity and a taxonomic correspondence to the type of organ (gut or hepatopancreas) (Li et al. 2019). Tzuc et al. (2014) isolated Vibrio strains from the shrimp stomach, hepatopancreas, and intestine (L. vannamei). These strains showed amylase and chitinase activities, which may be relevant in digestive processes. 
104 Still, additional research is required to understand the host-microbiome symbiosis and the 105 microbiome functions provided according to the colonized fraction in shrimp's digestive tract.

106 Therefore, the present work aimed to examine the microbiota variations along the gastrointestinal 107 tract of the Pacific white shrimp.

\section{Materials \& Methods}

110

\section{Bioassay: experimental design and sample collection}

An initial number of 350 white shrimp (Litopenaeus vannamei) in the postlarvae stage with a mean weight of $0.2 \mathrm{~g}$ were obtained from Cruz de Piedra aquaculture farm. After collection, healthy shrimp were acclimatized in a plastic container $(450 \mathrm{~L})$ for seven days with aerated filtered seawater $(35 \%)$, dissolved oxygen (DO) $\geq 5 \mathrm{mg} / \mathrm{L}$, temperature $30{ }^{\circ} \mathrm{C}$, water exchange $\left(25 \% \cdot\right.$ day $^{-}$ 1) and fed daily with a commercial diet.

116 Shrimp were randomly distributed into fifteen $80 \mathrm{~L}$ aquariums incorporated in a recirculation 117 system (RAS). Each aquarium contained 20 shrimp, weighing $0.5 \pm 0.1 \mathrm{~g}$. Before shrimp were introduced in the RAS, the experimental units were filled with sterile marine seawater to an operative volume of $60 \mathrm{~L}$. During the bioassay, the RAS's aquarium conditions maintained a

120 dissolved oxygen (DO) level of $5 \mathrm{mg} \cdot \mathrm{L}^{-1}$, salinity $35 \mathrm{UPS}$, and temperature $30{ }^{\circ} \mathrm{C}$. These parameters were daily measured using a YSI multiprobe system 556 (YSI Incorporated). in a closed circuit to reduce the variability of microenvironmental factors, generating the same 124 constant conditions in all aquariums. 
125 Further, the biofilter containing nitrifying bacteria maintained low concentrations of nitrogen

126 metabolites $\left(0 \mathrm{mg} \cdot \mathrm{L}^{-1}\right)$. Throughout the experiment, which lasted 80 days, shrimp were fed twice

127 a day with commercial feed (4\% biomass $\cdot$ day- 1$)$ consisting of $25 \%$ crude protein, $5 \%$ lipids, and

$1284 \%$ fiber. Also, uneaten feed, dead organisms, and molts were removed daily during the assay.

129 Prior to sampling, shrimp were fastened for $8 \mathrm{~h}$ to discard the transitory microbiota. A total of 75

130 individuals were chosen from the fifteen aquariums, i.e. five shrimp per aquarium were randomly

131 selected and pooled. Then, the digestive tract was aseptically dissected and divided into Foregut

132 (stomach), Midgut (hepatopancreas), and Hindgut (intestines). The samples (15 pools) were

133 individually placed in sterile tubes and stored at $-80^{\circ} \mathrm{C}$ until DNA isolation.

DNA isolation and 165 metagenomic sequencing

136 Genomic DNA was extracted using a commercial kit (FastDNA Spin Kit for Soil ${ }^{\mathrm{TM}}$; MP

137 Biomedicals, USA) according to the manufacturer's protocol and mechanically lysed and 138 homogenized using the FastPrep-24 ${ }^{\mathrm{TM}}$ 5G Instrument (MP Biomedicals ${ }^{\mathrm{TM}}$, USA). The DNA 139 concentration was determined using the Spectra Max fluorescence microplate reader (Molecular 140 Devices, USA) and the Quant-iT PicoGreen dsDNA assay kit (Invitrogen Molecular Probes Inc., 141 USA) to prepare the amplicon library according to the "16S-metagenomic sequencing library 142 preparation guide" published by Illumina. Finally, the targeted amplification of the 16S rRNA 143 gene V3 region was carried out.

144 In the case of the Midgut fraction, we only kept five pools $(\mathrm{n}=5)$ from the initially 15 pools because 145 some of the samples showed poor or no amplification. The difficulties of DNA extraction and 146 amplification from the Midgut fraction (hepatopancreas) are related to its high quantity of lipids 
147 and enzyme production that made complicated the DNA amplification (Carrillo-Farnés et al. 2007;

148 Cuzon et al. 2004; Schrader et al. 2012). On the other hand, 15 and 14 pools of the Hindgut and

149 Foregut fractions were adequate for the sequencing process.

150 The library was prepared by performing a PCR using primers flanking the hypervariable V3 region

151 of the bacterial 16S rRNA gene: 338- F (5'- ACTCCTACGGGAGGCAGCAG- 3') and 533-R (5'-

152 TTACCG CGGCTGCTG GCAC3') (Huse et al. 2008). All reactions, using 10 ng of DNA

153 template, were adjusted to $20 \mu \mathrm{l}$; then these were amplified by PCR $\left(95^{\circ} \mathrm{C}\right.$ for $3 \mathrm{~min}$, and 25 cycles

154 at $95^{\circ} \mathrm{C}$ for $30 \mathrm{~s}, 55^{\circ} \mathrm{C}$ for $30 \mathrm{~s}$, and $72^{\circ} \mathrm{C}$ for $30 \mathrm{~s}$, with a final extension at $72^{\circ} \mathrm{C}$ for $5 \mathrm{~min}$ ). A

155 second eight-cycle PCR $\left(95^{\circ} \mathrm{C}\right.$ x $30 \mathrm{~s} ; 61^{\circ} \mathrm{C}$ x $30 \mathrm{~s} ; 72^{\circ} \mathrm{C}$ x $\left.5 \mathrm{~min}\right)$ was performed to add Illumina

156 indexes, using Nextera XT Index Kit (Illumina, USA). The PCR amplicons were run through an

157 electrophoresis agarose gel, purified with Ampure XP magnetic beads (Beckman Coulter, USA),

158 and quantified using the Qubit 3.0 fluorometer (Thermo Fisher Scientific). Finally, an equimolar

159 pool $(4 \mathrm{nM})$ of the libraries was denatured and diluted to $2 \mathrm{pM}$. All the pool libraries were loaded

160 and sequenced using an Illumina Miniseq instrument (Illumina Biosystems, USA) using standard

161 conditions $(300$ cycles, $2 \times 150)$. All raw paired-end reads have been deposited in the Sequence

162 Read Archive (SRA) database at NCBI under BioProject ID PRJNA701069.

\section{Bioinformatics analyses}

164 Sequencing reads of the 16S rRNA gene were processed with Quantitative Insights Into Microbial 165 Ecology 2 (QIIME2) pipeline, version 2018.8 (Bolyen et al. 2018). For quality control, 166 demultiplex sequences were denoised, joined in paired reads, and chimeras were removed using 167 DADA2, a plugin of QIIME2 (Callahan et al. 2016). Based on sequence quality plots as a guide, 168 the first two nucleotides were trimmed, and the reads were truncated to 143 bases (Cicala et al. 169 2020). 
170 After removing low-quality scores, taxonomy was assigned to amplicon sequence variants (ASVs)

171 using a pre-trained classifier Silva_132 with OTUs clustered at 99\%. Unassigned sequences and

172 low confidence $(0.005 \%)$ ASVs were removed, meaning that ASVs with frequency $<4$ reads were

173 discarded before further analyses (Cornejo-Granados et al. 2018).

174 A rooted phylogenetic tree was generated for microbial diversity analyses. Multiple sequence

175 alignment of ASV representative sequences was performed using MAFFT software (Katoh \&

176 Standley 2013). Further, FastTree (Price et al. 2010) software was used for building a phylogenetic

177 tree, which shows the relationships of different bacterial species in a tree-like model that includes

178 nodes.

179

180 Data analysis and statistics

181 Library sizes were adjusted, rarefying the number of reads to a subsampled minimum depth of

1825098 reads to avoid unequal sample sizes and estimated alpha and beta diversity. A rarefaction

183 curve was generated using ASVs to estimate species richness (alpha diversity) with the qiime

184 diversity alpha-rarefaction plugin implement in QIIME2 (Bolyen et al. 2018). Also, alpha diversity

185 was calculated using Chaol and Shannon index and Observed ASV among the different digestive

186 tract fractions. The raw count of ASVs and ASVs clustered at the family level were used to

187 calculate the indexes. A Kruskal and Wilcoxon statistic test $(\mathrm{p}<0.05)$ was performed in the raw

188 ASV set. Index comparison at the family level, a one-way ANOVA, and a Tukey statistical test (p

$189<0.05)$ was used for the multiple pairwise comparisons among the digestive tract fractions/tissues

190 To evaluate the microbiome community structure, the compositional similarity/dissimilarity

191 between samples (beta diversity) was estimated by generating weighted UniFrac, Jaccard, and 
192 Bray-Curtis dissimilarity matrices. The dissimilarity between fraction samples was visualized

193 using principal coordinate analysis (PCoA) (all listed above performed using QIIME2 diversity

194 plugin). Pairwise comparison of the digestive tract beta diversity distances was performed using

195 permutation multivariate analysis of variance (PERMANOVA) through 4,999 permutations with

196 a p-value of 0.0002 to the beta diversity analysis of QIIME.

197 To identify the significant taxa in the digestive tract fractions, we also test a Differential

198 Abundance (DA) analysis to identify the significant taxa in the digestive tract fractions. A linear

199 regression framework carried out the multigroup differential abundance analysis with Bias

200 correction for the unobservable differential sampling fractions across samples (i.e., sampling

201 fraction of a sample drawn from a unit volume of an ecosystem) (Lin \& Peddada 2020). According

202 to the fraction co-variability: Midgut $(n=5)$, Hindgut $(n=15)$, and Foregut $(n=14)$, the feature

203 table was agglomerated at the family level. The DA analysis uses the absolute abundance of

204 intersected families' microbiome data in the digestive tract fractions (76 families, then). Here, we

205 used prevalence to filter the absolute abundance of microbiome data as a form of unsupervised

206 filtration (total abundance $>1 \%$ ). Since filtering was unsupervised upon the taxonomic

207 annotations, the undetermined families were labeled with their last incomplete lineages. In this

208 case, we assumed that the features (before filtering steps in the bioinformatic workflow, such as

209 chimeric removal, quality read filtering, low filtering features, etcetera) represent a combination

210 of real biological variants that remain insufficiently documented due to a lack of representatives

211 in the reference database (Hibbett \& Glotzer 2011). Finally, an occurrence-based visualization was

212 performed to show the intersection of the number of families through the three digestive tract

213 fractions (Conway et al. 2017). 
214 In addition to the occurrence-based visualization, we quantified the intra-order interaction of

215 covariates by using non-binary co-expression networks analysis (Weighted topological, wTO).

216 The wTO method measures a link-to-link threshold of significance p adjusted $\left(\mathrm{p}_{\text {adj }}\right)$ value $<0.01$

217 (p-values, adjusted for multiple testing). Here, positive correlations (blue color links) may

218 represent symbiotic or commensal relationships, while negative correlations (red color links) may

219 represent predator-prey interactions, allelopathy, or competition for limited resources (Gysi et al. 220 2018).

\section{Functional metagenomic prediction}

223 A PICRUSt2 pipeline was used for functional metagenomic prediction (Douglas et al. 2020). The

224 files of ASVs abundance table and represented sequences were also used as an input to assess

225 functional predictions and matching ASVs normalized by $16 \mathrm{~S}$ rRNA copy number against the 226 Kyoto Encyclopedia of Genes and Genomes (KEGG) database. The implemented commands were 227 place_seqs.py, hsp.py, and metagenome_pipeline.py to create the predicted metagenomics table.

228 Finally, the Statistical Analysis of Metagenomic Profiles (STAMP) software v2.1.3 was used to 229 analyze the metagenomes predicted by PICRUSt2. Significant differences in the KEGG pathways 230 between the microbiota from the digestive tract fractions were monitored using the non-parametric 231 Kruskal-Wallis test and Bonferroni approach for multiple test correction, and a $\mathrm{p}<0.05$ was 232 considered significant.

\section{Results}


236 A total of $1,609,904$ 16S rRNA raw reads were generated during sequencing. Quality control

237 analysis returned 1,086,676 sequences (67.49\%) that were clustered into 614 ASVs. The

238 rarefaction curves showed that the microbial community was correctly represented by the

239 sequencing data (Fig. S1). Alpha diversity was calculated using Chao1 and Shannon index and

240 Observed ASV among the different digestive tract fractions using two ASVs sets. Regarding the

241 raw ASVs count, no significant differences were detected $(\mathrm{p}<0.05)$. When the ASVs were

242 clustered to the family level, only the Shannon diversity Index showed differences $(p<0.05)$

243 between Hindgut-Midgut and Foregut-Midgut comparisons (Fig. 1).

244 In order to test differential groups of taxa, the feature table was agglomerated at the family level,

245 using prevalence filtering as a form of unsupervised filtration. Prevalence analysis reported that

246 Proteobacteria had the highest spread, followed by Actinobacteria, Verrucomicrobia, Firmicutes,

247 Bacteroidetes, and Cyanobacteria (Fig. S2). A whole set of 577 features were retained after this

248 filtration step (180,879 [0.996\%] absolute abundance). A set of 31 families was intersected

249 through the three fractions; 33, 7, and 5 intersections were found in Hindgut-Foregut, Midgut-

250 Foregut, and Hindgut-Midgut, respectively. A set of families were exclusive per fraction: Midgut

251 (15), Foregut (17), and Hindgut (19). Families presented only in a single fraction were excluded 252 from the DA test (Fig. 2).

254 Analysis of the taxonomic profile

255 A barplot was performed with the relative bacterial abundance at the phylum level for each fraction

256 (Fig. 3). The ASV abundance was variable across the three digestive tract fractions. Here,

257 Actinobacteria, Bacteroidetes, Firmicutes, and Proteobacteria were represented in the three 
258 digestive tract fractions. Particularly, Proteobacteria was the enriched phyla in all the fractions,

259 whereas Actinobacteria was enriched in the Hindgut fraction. Conversely, Verrumicrobia was

260 exclusive in either fractions, Foregut and Hindgut tissues. The results obtained with the DA test

261 showed a similar pattern in the shared and exclusive taxa groups presented in the three digestive

262 tract fractions.

263 Additionally, a relative abundance bar plot with the DA families (significance $<0.05$ ) across the

264 digestive tract fractions was performed. The bar plot (Fig. 4) shows a high percentage of classified

265 ASVs belonging to the Rhodobacteraceae (74\%) and Rubritalaceae (80\%) in the Foregut fraction.

266 Also, Cellulomonadaceae (73\%) was an enriched family, particularly in the Hindgut. The

267 Aeromonadaceae (90\%) and Beijerickiaceae (88\%) families registered a great abundance range in 268 the Midgut fraction.

\section{Bacterial community structure of the digestive tract fractions}

271 PCoA analysis revealed significant differences among fractions from the digestive $\operatorname{tract}(\mathrm{p}<0.05)$.

272 Figure 5 shows the PCoA of beta diversity associated with microbiota variance for Foregut 273 (stomach), Midgut (hepatopancreas), and Hindgut (intestine), using Weighted UniFrac (A), 274 Jaccard (B), and Bray Curtis (C) distances. Beta diversity metrics were evaluated with 275 PERMANOVA with 4999 permutations with a p-value of 0.0002. Weighted UniFrac metrics (Fig. $2765 \mathrm{~A}$ ) registered $72.67 \%$ of the total variance. On the contrary, the principal components of Jaccard 277 (Fig. 5B) represented an accumulated variance of 27.3\%, while Bray-Curtis clustering (Fig. 5C) 278 showed the highest value of 53\% of the total variability (PC1 24\%, PC2 18\%, and PC3 11\%). 279 Despite total variability differences among PCoA, microbial beta-diversity's community structure 
280 using all the metrics presented three primary groups for each organ where Hindgut and Foregut

281 registered a more remarkable similarity.

\section{Differences in the microbial abundance from the digestive tract fractions}

284 The differential abundance (DA) analysis with the analysis composition of microbiomes with bias correction was performed to identify significant family abundance differences among the digestive tract fractions (Fig. 6). Furthermore, the number of families observed in the shrimp Foregut (stomach), Midgut, and Hindgut var according to the phylum and fraction. For instance, sixteen significant families were observed from the phylum Proteobacteria. In the case of the phylum Actinobacteria, five significant families were detected. Verrumicrobia registered only one family, Firmicutes three families, and Bacteroidetes two families.

The DA analysis compares the family abundance between Foregut, Midgut, and Hindgut fractions. In this regard, the microbial communities' abundance varied considerably depending on the digestive tract fraction. In the Foregut fraction, the Rhodobacteraceae and Rubritalaceae families are significantly more abundant than in the Midgut and Hindgut. Otherwise, in the Midgut fraction, three families (Beijerinckiaceae, Aeromonadaceae, and Propionibacteriaceae) were significantly higher in terms of abundance than in the other two digestive tract fractions. However, other families registered differences in the Midgut fraction against Hindgut or Foregut. For instance, Micrococcaceae registered a greater abundance in the Midgut, with significant differences only against|the Hindgut. Finally, the Cellulomonadaceae family resulted in a high proportion in the was significantly superior in the Foregut and Hindgut in comparison with the Midgut fraction. 


\section{Bacterial interactions at order level using wTO}

304

305

306

307

308

309

310

311

312

313

314

315

316

317

318

319

320

321

322

323

324

In the weighted topological (wTO) network analysis, we found cumulative features at order level, with at least one significant interaction: 37 interactions were found in the Hindgut (44 Orders), 8 interactions in the Midgut (34 Orders), and 34 in Foregut fraction (43 Orders) at 99\% confidence $\left(\mathrm{p}_{\text {adj-value }}<0.01\right)$. There were five clusters in the Foregut fraction (Fig. 7), although the interactions of cluster 1 and 2 showed great strength, there was greater "shade" (which represent how strong the interaction is) and a more significant contribution in the cluster 5; especially the order Pseudomonadales seems to be the "key" for the connection or communication to the order Bacillales and Lactobacillales. On the other hand, Micrococcales and Cellvibrionales were negatively correlated with Pseudomonadales, Propionibacteriales, Cellvibrionales. The interaction network in the Midgut was poor, maybe related to the sample number (Fig. 8). In this fraction, we

found two clusters; cluster 1, showed a network only with the Proteobacteria phylum in equal contribution. On the other hand, Propionibacteriales in cluster 2 was the center of the cluster and the gate of interaction from a more diverse microbiota, such as Desulfobacterales and Cyptophagales. Finally, the Hindgut fraction interactions (Fig. 9) were highly different from the other two fractions. Four clusters were in the Hindgut. Positive interactions were mainly found in all the clusters, except for cluster 4, which suggests a set of bacterial species (Lactobacillales and Vibrionales) that do not coexist with other bacteria from Cluster 2 and 3 . The Costridiales order in Cluster 3 contributed with the highest number of microorganisms, as well as the Rhizobiales. These seem to be directly antagonists to the order Vibrionales. 
325 Bacterial metabolic functions from digestive tract fractions were enriched and predicted based on

326 KEGG. The Nearest Sequenced Taxon Index (NSTI) was estimated and calculated per sample,

327 and 10 of 900 ASVs resulting above the max NSTI cut-off of 2.0 were removed.

328 A heatmap was performed of the KEGG predicted pathways across all the digestive tract fractions

329 (Fig. 10). The three principal predicted functions with greater abundance in the digestive fractions

330 studied were related to Metabolism (74\%), Genetic Information Processing (14.8\%), and Cellular

331 Processes (4.05\%). Specifically, in the Foregut, the enriched pathways were related to Metabolism

332 and Cellular Processes. While in the Midgut, besides metabolism, pathways related to genetic

333 information processing and organismal systems were also registered. Likewise, the Hindgut also

334 registered Metabolism and Genetic information processing predicted pathways and Environmental

335 Information Processing. This last pathway was only enriched in this digestive tract fraction.

336 Also, differentially abundant predicted functional features between the digestive tract fractions

337 were compared and analyzed using STAMP. A total of 27 KEGG pathways with significant

338 differences $(\mathrm{p}<0.05)$ were detected based on the Kruskal-Wallis test. As shown in Table 1,

339 regarding metabolism, the enriched subcategories level-2 pathways in the Foregut were Amino

340 acid metabolism, Carbohydrate Metabolism, Energy metabolism, and Xenobiotics biodegradation

341 and metabolism. Notably, Carbohydrate metabolism (C5-Branched dibasic acid metabolism and

342 Glyoxylate and dicarboxylate metabolism) was significantly higher in this digestive tract fraction.

343 The cellular processes predicted pathway was also found in this fraction, with genes involved in

344 the cell growth pathway such as apoptosis. In particular, this level-3 of the functional subcategory

345 was significant in the Foregut.

346 Likewise, in the Midgut fraction, the enriched metabolism pathways included Amino acid

347 metabolism (Tryptophan metabolism), Metabolism of cofactors and vitamins (Folate biosynthesis 
348 and Vitamin B6 metabolism), Metabolism of terpenoids and polyketides (Carotenoid

349 biosynthesis), Xenobiotics biodegradation and metabolism (Toluene degradation), and Lipid

350 metabolism. Interestingly, bacterial communities from the Midgut fraction had a significantly

351 greater abundance of Lipid metabolism, with the level-3 subcategory glycerophospholipid

352 metabolism pathway and metabolism of terpenoids and polyketides such as carotenoid

353 biosynthesis.

354 In contrast, five predicted pathways were enriched in the Hindgut fraction. Metabolism of

355 cofactors and vitamins (Nicotinate and nicotinamide metabolism), Energy metabolism (Nitrogen

356 metabolism), and Xenobiotics biodegradation and metabolism were the metabolic pathways

357 enriched. Other enriched functions included Environmental information processing such as

358 Membrane transport and metabolism (Phosphotransferase system) and Genetic information

359 processing that includes the Replication and repair pathway.

Distances of the predicted bacterial functions among the digestive tract fractions in a principal component analysis (PCOA)

A principal component analysis (PCoA) was performed on the relative abundance values of the

KEGG pathways of the digestive tract microbiota. The PCoA showed a clear distinction between the clustering on the predicted functions from the microbiota of the digestive tract's three fractions. The predicted functional profile showed significant differences among the fractions, based on the Kruskal-Wallis test and the Bonferroni approach for multiple test correction using STAMP. Foregut and shared few functions with the intestinal microbiota (Fig. S3). 


\section{Discussion}

372 The shrimp's gut microbial ecosystem is a whole essential system playing vital symbiotic roles in

373 maintaining homeostatic physiology in the host. However, most studies about crustacean

374 microbiota have been exclusively focused on the intestine (Cornejo-Granados et al. 2018; Cheung

375 et al. 2015). This study provides an approach to the entire digestive tract, including the Foregut

376 (stomach), Midgut (hepatopancreas), and Hindgut (intestine) fractions.

377 The relative abundance bar plot with the DA families revealed that, at the family level,

378 Rhodobacteracea was well represented in the Foregut, while in the Midgut was underrepresented.

379 Liu et al. (2019) reported that Rhodobacteraceae could be potentially applied to reduce the

380 influence of cold stress in white shrimp; however, the members of Rhodobacteraceae have diverse

381 physiological and metabolic characteristics and are often found in marine animals as symbiotic

382 bacteria (Pujalte et al. 2014). Besides, Rhodobacteraceae bacteria seem to exhibit probiotic

383 potential due to their ability to produce tropodithietic acid (TDA) to inhibit pathogens. These can

384 also synthesize vitamin B12, which is essential for shrimp growth (Sañudo-Wilhelmy et al. 2014).

385 Notably, this family was mainly found in the Foregut fraction. This fraction consists of a stomach

386 connected to an esophagus with further divisions (cardiac chamber and pyloric chamber)

387 (Muhammad et al. 2012). Mouthparts perform food particles' intake; food passes the esophagus

388 and reaches the stomach for mechanical and extracellular digestion (McGaw \& Curtis 2013). The

389 high abundance of Rhodobacteraceae could be associated with its action against pathogenic

390 bacteria as a first defense barrier at the beginning of the digestive tract required by the host

391 (Soonthornchai et al. 2015). 
392 On the other hand, the Cellulomonadaceae family was mainly found in the shrimp Hindgut, and

393 this family has been reported in shrimp ponds (Wang et al. 2017a). A large variety of hydrolytic

394 starch, xylan, and cellulose-degrading enzymes, are produced by Cellulomonadaceae, which

395 belongs to the Actinobacteria phylum. Short-chain fatty acids, such as acetate, are produced by

396 some Actinobacteria that promote the host epithelial cells' defense functions and protect the host

397 from lethal infections (Fukuda et al. 2012). Specifically, genera from this family, like Actinotalea

398 can degrade cellulose and produce acetate (Stackebrandt \& Schumann 2014), which has high

399 inhibitory activity against pathogenic Vibrio species in L. vannamei (da Silva et al. 2013).

400 The family Beijerinckiaceae was only detected in the Midgut (hepatopancreas). It belongs to the

401 order Rhizobiales within the phylum Alphaproteobacteria; the main genera represented in this

402 family was Methlylobacterium. This group encompasses aerobic bacteria capable of forming poly-

$403 \beta$-hydroxybutyrate granules and fixing nitrogen; furthermore, they can increase exopolysaccharide

404 production (Marín \& Arahal 2014). Fixing nitrogen bacteria in aquatic organisms as symbionts

405 have been poorly studied; however, nitrogen fixation processes within the microbial community

406 from the digestive tract of teleost species, like the catfish, Panaque nigrolineatus, have been

407 reported (McDonald et al. 2015). According to previous reports performed by Lu et al. (2016),

408 there are genes and pathways involved in nitrogen metabolism that play an essential role in

409 reducing ammonia toxicity in the hepatopancreas of L. vannamei; also, ammonia stress could

410 inhibit the immune system and increase the susceptibility of shrimp to pathogens. Overall, it seems

411 that the Midgut gland provides a suitable niche for nitrogen fixation that may facilitate the

412 production of reduced nitrogen by the Beijeranckiaceae family.

413 The microbial communities from the digestive tract's fractions showed that shrimp gut microbiota

414 varies depending on the fraction sampled. Comparisons of beta diversity Bray-Curtis distances 
415 showed consistent separation between the Foregut and Hindgut against the Midgut gland,

416 suggesting that the white shrimp could provide a unique ecological niche according to their

417 digestive tract fraction.

418 Nevertheless, there were families shared between the digestive tract fractions. Families across the 419 digestive tract showed a set of 31 groups through the three digestive fractions. However, the 420 analysis exhibited that the Foregut and Hindgut were the fractions sharing a higher number of

421 families (33), unlike Midgut-Foregut (7) and Hindgut-Midgut (5). The more significant number of 422 families shared among Foregut and Hindgut is possibly due to the niche structure's similarity.

423 Cheung et al. (2015) reported no apparent differentiation between bacterial communities in the 424 Foregut and the Hindgut of the crustacean Neocaridina denticulata because both present a 425 chitinous lining despite the distinct physiological functions of these organs. However, the 426 attachment of bacteria could also be associated with the host's functional requirements in the 427 Midgut fraction. For example, in other crustaceans, endosymbiotic bacteria contributing to 428 digestive processes thrive in this digestive tract fraction (Zimmer et al. 2001). However, other 429 omics approaches are required to understand how these differences between the gastrointestinal 430 fractions contribute to the symbiotic relationship.

431 Highly complex interactions were observed in the gut microbiota fractions. In the Hindgut, the gut 432 network was mainly focused on the order Costridiales and Rhizobiales, which could strongly 433 inhibit Vibrionales. A possible explanation of this networking is that these could compete for 434 nutrient resources or adhesion sites, displacing the order Vibrionales. Besides, the order 435 Costridales is a Gram-positive butyric acid-producing probiotic, Clostridium butyricum, which is 436 also described as part of the normal intestine microbiota of shrimp and has a beneficial effect on 437 the intestine health of L. vannamei (Duan et al. 2018). Also, the order Rhizobiales has already been 
438 reported to exert an antagonistic effect against Vibrionales in postlarval shrimp (L. vannamei) (Cao

439 et al. 2020). Some vibrios (as Vibrionales) are recognized as opportunistic pathogens causing

440 vibriosis in shrimp (Chandrakala \& Priya 2017); however, the above antagonistic network (and

441 others still unknown) could explain the presence of pathogenic strains without producing harmful

442 effects in shrimp.

443 Along with the taxonomic profile, this approach provides insight into the gut microbiota's

444 functions. In the long-term, the predicted functional profiles from the digestive tract fractions

445 exhibited differences. We found that the digestive tract microbiota could play a key role in host

446 nutrition. In this study, the differences in predictive functional pathways between the digestive

447 tract fractions were based on their microbial composition and functional capabilities. Our results

448 indicate that genes involved in Carbohydrate Metabolism showed greater abundance in the Foregut

449 fraction. In this regard, shrimp has a limited capacity to metabolize nutrients like carbohydrates;

450 alternatively, shrimp uses proteins as a source of energy and growth (Rosas et al. 2000). However,

451 the harbored microbiota presents Carbohydrate Metabolism (Glyoxylate and dicarboxylate

452 metabolism), probably contributing with metabolic power to cope with the host's deficiencies. Ma

453 et al. (2020) reported a relationship between carbohydrate metabolism pathways and the Vibrio

454 harveyi infection's progress due to the high energy demand for shrimp survival (Wang et al.

455 2017b).

456 The hepatopancreas is the main secretory organ of shrimp and the primary absorption barrier of 457 crustaceans' nutrients (Jobling 2012). Besides, it is the main organ of lipid metabolism. In this 458 regard, the Lipid metabolism (Glycerophospholipid metabolism) pathway and the metabolism of 459 terpenoids and polyketides such as carotenoid biosynthesis were representatives of the Midgut 460 fraction. Enzymatic activity from shrimp digestive tract microbiota has already been reported; 
461 specifically, Vibrio and Pseudoalteromonas strains isolated from hepatopancreas, presented 462 amylase, chitinase, lipase, and esterase activities, indicating that L. vannamei microbiota includes 463 some groups exhibiting multi enzymatic activity that contributes to the degradation of dietary 464 components (Tzuc et al. 2014).

465 Also, the hepatopancreas is an important indicator of crustaceans' host health condition (Deng et 466 al. 2017). Previous studies of the crayfish's (Procambarus clarkia) hepatopancreatic microbiota 467 revealed that diseased organisms underwent dysbiosis in the gut-hepatopancreatic microbiota, 468 affecting lipid synthesis pathways (Wu et al. 2021). Although, the relationship between gut 469 dysbiosis and physiological effects in lipid synthesis remains unknown.

470 Lipids are essential in aquatic animals to reduce osmotic shock due to preserving the ion balance 471 and regulating biological membranes' structure. Glycerophospholipids, glycerol-based 472 phospholipids, are the main biological membranes component (Chen et al. 2014; Palacios et al. 473 2004). In the study reported by Chen et al. (2015) lipid metabolism is involved in osmoregulation 474 strategies in essential organs in the white shrimp.

475 Not only the Lipid metabolism pathway was enriched in the Midgut but also Carotenoid 476 biosynthesis. Carotenoids are lipid-soluble tetraterpenoid compounds, and, in general, animals are 477 cannot biosynthesize these compounds. Plants and microorganisms like bacteria primarily 478 biosynthesize these. However, carotenoids are essential antioxidants and play crucial functions in 479 health (Tanumihardjo 2012). Additionally, they are natural pigments involved in animals' body 480 coloration; for instance, astaxanthin is a carotenoid largely responsible for the color of shrimp, and 481 diets for shrimp aquaculture are added with this carotenoid to improve its visual appeal (Su et al. 482 2018). 
483 Pathways related to the metabolism of cofactors and vitamins (Nicotinate and nicotinamide 484 metabolism) were more abundant in the Hindgut microbiota than other digestive segments.

485 Previously there are reports of microbial sequences annotated to energy metabolism, xenobiotics 486 biodegradation, membrane transport in intestinal microbiota from two cultured stages $(30 \mathrm{~d}$ and $48760 \mathrm{~d}$ ) of L. vannamei (Gao et al. 2019), even so, their implications in the intestine shrimp are not 488 yet elucidated. However, regarding energy metabolism, such as nitrogen metabolism, Ortiz489 Estrada et al. (2021) reported genes encoding enzymes implicated in the nitrogen metabolism in 490 probiotic biofilm from a zero recharge shrimp culture, which registered better water quality 491 parameters than a heterotrophic biofilm. Nitrogenous metabolites, particularly unionized ammonia $492\left(\mathrm{NH}_{3}\right)$ and nitrite $\left(\mathrm{NO}_{2}\right)$ are toxic for shrimp. Consequently, the ideal condition requires the rapid 493 oxidation of ammonia/ammonium to nitrite and, then, to nitrate. Such processes are carried out in 494 aerobic environments by ammonia-oxidizing bacteria and nitrite-oxidizing bacteria (Robles495 Porchas et al. 2020). On the whole, Metabolic predicted functions in the digestive tract fractions 496 are necessary for adequate host development.

497 Finally, the taxonomic and predicted functional differences among gut fractions suggest that these 498 could provide specialized functions for each organ. As stated above, the association of diseases with dysbiosis in organs, like the intestine and the hepatopancreas in some crustaceans, indicates 500 that the microbiota can play a protective role against pathogens in the different gut fractions.

501 However, these microbiotas' communication with the crustacean systems is still hypothetical and 502 should be addressed in further approaches. 
505 The three different digestive tract compartments showed that bacterial populations varied

506 substantially between the shrimp gut regions, especially between the Midgut (hepatopancreas) and

507 Hindgut (intestine). The Midgut (hepatopancreas) seems to be a fraction harboring exclusive

508 microbiota since it was the tissue with less diversity and less vulnerable to its microbiota changes.

509 Not only the microbiota shifted in accordance with the digestive tract component, but also their

510 predicted functions. In the Foregut, Carbohydrate metabolism was mainly registered in this

511 fraction. On the contrary, pathways related to Lipid metabolism prevailed in the Midgut. In the

512 Hindgut, pathways like the metabolism of cofactors and vitamins together with energy metabolism

513 were enriched in this fraction. Finally, lipid metabolism pathways seem to be exclusively and

514 essential in the Midgut. According to host physiology, the present study contributed to

515 understanding the differences in microbiota composition harbored by the different fractions of

516 shrimp gut, providing a basis for studying the microbiota's role in the different gut fractions.

517

\section{Acknowledgments}

519 The authors would like to acknowledge the Mexican Government through the National Council of

520 Science and Technology (CONACyT) for the Ph.D. fellowship granted. 


\section{Declarations:}

523

524 Funding:

525 Not applicable

526 Conflicts of interest/ Competing interests:

527 The authors declare no conflict of interest

528 Availability of data and material:

529 Data will be available upon request. Metagenomics data can be found in the database indicated

530 Code availability:

531 Not applicable

\section{Ethics approval:}

533 Not applicable

\section{Authors' contributions}

535 Estefanía Garibay-Valdez: Experiment, Bioinformatics, Data analysis, Manuscript writing,

536 Revision and edition of the final draft

537 Marcel Martínez-Porchas: Manuscript conception, Experiment, Bioinformatics, Data analysis,

538 Manuscript writing, Supervision, Revision and edition of the final draft

539 Francesco Cicala: Experiment, Bioinformatics, Data analysis, Manuscript writing

540 Gómez-Reyes Ricardo: Bioinformatics 
541 Francisco Vargas-Albores: Data analysis, Bioinformatics, Manuscript writing

542 Teresa Gollas-Galván: Data analysis, Manuscript writing

543 Luis Rafael Martínez-Córdova: Data analysis, Revision and edition of the final draft

544 Kadiya Calderón: Revision and edition of the final draft

545 
546

547

548

549

550

551

552

553

554

555

556

557

558

559

560

561

562

563

564

565

566

567

568

569

570

571

572

573

574

575

576

577

578

579

580

581

582

583

584

585

586

587

588

589

590

591

\section{References}

Belkaid Y, and Hand TW. 2014. Role of the microbiota in immunity and inflammation. Cell 157:121-141. https://doi.org/10.1016/i.cell.2014.03.011

Bolyen E, Rideout JR, Dillon MR, Bokulich NA, Abnet C, Al-Ghalith GA, Alexander H, Alm EJ, Arumugam $M$, and Asnicar F. 2018. QIIME 2: Reproducible, interactive, scalable, and extensible microbiome data science.

Brune A, and Dietrich C. 2015. The Gut Microbiota of Termites: Digesting the Diversity in the Light of Ecology and Evolution. Annual Review of Microbiology 69:145-166. 10.1146/annurev-micro092412-155715

Callahan BJ, McMurdie PJ, Rosen MJ, Han AW, Johnson AJA, and Holmes SP. 2016. DADA2: highresolution sample inference from Illumina amplicon data. Nature methods 13:581-583.

Cao Q, Najnine F, Han H, Wu B, and Cai J. 2020. BALOs Improved Gut Microbiota Health in Postlarval Shrimp (Litopenaeus vannamei) After Being Subjected to Salinity Reduction Treatment. Frontiers in Microbiology 11. https://doi.org/10.3389/fmicb.2020.01296

Carrillo-Farnés O, Vega-Villasante F, Forrellat-Barrios A, and Guerrero-Galván S. 2007. A review of digestive enzyme activity in penaeid shrimps. Crustaceana 80:257-275.

Cicala F, Lago-Lestón A, Gomez-Gil B, Gollas-Galván T, Chong-Robles J, Cortés-Jacinto E, and MartínezPorchas M. 2020. Gut microbiota shifts in the giant tiger shrimp, Penaeus monodon, during the postlarvae, juvenile, and adult stages. Aquaculture International 28:1421-1433. https://doi.org/10.1007/s10499-020-00532-1

Conway JR, Lex A, and Gehlenborg N. 2017. UpSetR: an R package for the visualization of intersecting sets and their properties. Bioinformatics 33:2938-2940.

Cornejo-Granados F, Gallardo-Becerra L, Leonardo-Reza M, Ochoa-Romo JP, and Ochoa-Leyva A. 2018. A meta-analysis reveals the environmental and host factors shaping the structure and function of the shrimp microbiota. PeerJ 6:e5382.

Cornejo-Granados F, Lopez-Zavala AA, Gallardo-Becerra L, Mendoza-Vargas A, Sánchez F, Vichido R, Brieba LG, Viana MT, Sotelo-Mundo RR, and Ochoa-Leyva A. 2017. Microbiome of pacific whiteleg shrimp reveals differential bacterial community composition between wild, aquacultured and AHPND/EMS outbreak conditions. Scientific Reports 7:11783. https://doi.org/10.1038/s41598-017-11805-w

Cuzon G, Lawrence A, Gaxiola G, Rosas C, and Guillaume J. 2004. Nutrition of Litopenaeus vannamei reared in tanks or in ponds. Aquaculture 235:513-551.

Chandrakala N, and Priya S. 2017. Vibriosis in shrimp aquaculture a review. Int J Scientif Res Sci 3:27-33.

Chen K, Li E, Gan L, Wang X, Xu C, Lin H, Qin JG, and Chen L. 2014. Growth and lipid metabolism of the pacific white shrimp Litopenaeus vannamei at different salinities. Journal of Shellfish Research 33:825-832.

Chen K, Li E, Li T, Xu C, Wang X, Lin H, Qin JG, and Chen L. 2015. Transcriptome and molecular pathway analysis of the hepatopancreas in the pacific white shrimp Litopenaeus vannamei under chronic low-salinity stress. PloS one 10:e0131503. https://doi.org/10.1371/journal.pone.0131503

Cheung MK, Yip HY, Nong W, Law PTW, Chu KH, Kwan HS, and Hui JHL. 2015. Rapid change of microbiota diversity in the gut but not the hepatopancreas during gonadal development of the new shrimp model Neocaridina denticulata. Marine Biotechnology 17:811-819.

https://doi.org/10.1007/s10126-015-9662-8

da Silva BC, do Nascimento Vieira F, Mouriño JLP, Ferreira GS, and Seiffert WQ. 2013. Salts of organic acids selection by multiple characteristics for marine shrimp nutrition. Aquaculture 384:104-110. 
592

593

594

595

596

597

598

599

600

601

602

603

604

605

606

607

608

609

610

611

612

613

614

615

616

617

618

619

620

621

622

623

624

625

626

627

628

629

630

631

632

633

634

635

636

637

638
Dai W, Sheng Z, Chen J, and Xiong J. 2020. Shrimp disease progression increases the gut bacterial network complexity and abundances of keystone taxa. Aquaculture 517:734802.

https://doi.org/10.1016/j.aquaculture.2019.734802

Das S, Ward LR, and Burke C. 2008. Prospects of using marine actinobacteria as probiotics in aquaculture. Applied microbiology and biotechnology 81:419-429. https://doi.org/10.1007/s00253-008-1731-8

Deng Y, Wang Y, Zhang X, Sun L, Wu C, Shi Q, Wang R, Sun X, Bi S, and Gooneratne R. 2017. Effects of T-2 toxin on Pacific white shrimp Litopenaeus vannamei: growth, and antioxidant defenses and capacity and histopathology in the hepatopancreas. Journal of aquatic animal health 29:15-25.

Douglas GM, Maffei VJ, Zaneveld J, Yurgel SN, Brown JR, Taylor CM, Huttenhower C, and Langille MG. 2020. PICRUSt2: An improved and customizable approach for metagenome inference. BioRxiv:672295.

Duan Y, Wang Y, Dong H, Ding X, Liu Q, Li H, Zhang J, and Xiong D. 2018. Changes in the Intestine Microbial, Digestive, and Immune-Related Genes of Litopenaeus vannamei in Response to Dietary Probiotic Clostridium butyricum Supplementation. Frontiers in Microbiology 9. https://doi.org/10.3389/fmicb.2018.02191

Fukuda S, Toh H, Taylor TD, Ohno H, and Hattori M. 2012. Acetate-producing bifidobacteria protect the host from enteropathogenic infection via carbohydrate transporters. Gut Microbes 3:449-454. https://doi.org/10.4161/gmic.21214

Gao S, Pan L, Huang F, Song M, Tian C, and Zhang M. 2019. Metagenomic insights into the structure and function of intestinal microbiota of the farmed Pacific white shrimp (Litopenaeus vannamei). Aquaculture 499:109-118. https://doi.org/10.1016/j.aquaculture.2018.09.026

Garibay-Valdez E, Martínez-Porchas M, Calderon K, Vargas-Albores F, Gollas-Galván T, and MartínezCórdova L. 2020. Taxonomic and functional changes in the microbiota of the white shrimp (Litopenaeus vannamei) associated with postlarval ontogenetic development. Aquaculture 518:734842.

Gómez GD, and Balcázar JL. 2008. A review on the interactions between gut microbiota and innate immunity of fish. FEMS Immunology \& Medical Microbiology 52:145-154. https://doi.org/10.1111/j.1574-695X.2007.00343.x

Gysi DM, Voigt A, Fragoso TdM, Almaas E, and Nowick K. 2018. wTO: an R package for computing weighted topological overlap and a consensus network with integrated visualization tool. $B M C$ Bioinformatics 19:392. https://doi.org/10.1186/s12859-018-2351-7

Hibbett D, and Glotzer D. 2011. Where are all the undocumented fungal species? A study of Mortierella demonstrates the need for sequence-based classification. New Phytologist 191:592-596.

Hossain MS, Dai J, and Qiu D. 2021. Dysbiosis of the shrimp (Penaeus monodon) gut microbiome with AHPND outbreaks revealed by $16 \mathrm{~S}$ rRNA metagenomics analysis. Aquaculture Research. doi.org/10.1111/are.15178

Huang Z, Zeng S, Xiong J, Hou D, Zhou R, Xing C, Wei D, Deng X, Yu L, and Wang H. 2020a. Microecological Koch's postulates reveal that intestinal microbiota dysbiosis contributes to shrimp white feces syndrome. Microbiome 8:1-13.

Huang Z, Zeng S, Xiong J, Hou D, Zhou R, Xing C, Wei D, Deng X, Yu L, Wang H, Deng Z, Weng S, Kriengkrai S, Ning D, Zhou J, and He J. 2020b. Microecological Koch's postulates reveal that intestinal microbiota dysbiosis contributes to shrimp white feces syndrome. Microbiome 8:32. https://doi.org/10.1186/s40168-020-00802-3

Huse SM, Dethlefsen L, Huber JA, Welch DM, Relman DA, and Sogin ML. 2008. Exploring Microbial Diversity and Taxonomy Using SSU rRNA Hypervariable Tag Sequencing. PLOS Genetics 4:e1000255. 10.1371/journal.pgen.1000255

Peer) reviewing PDF | (2021:03:59686:2:0:NEW 23 Jun 2021) 
639

640

641

642

643

644

645

646

647

648

649

650

651

652

653

654

655

656

657

658

659

660

661

662

663

664

665

666

667

668

669

670

671

672

673

674

675

676

677

678

679

680

681

682

683

684

685

686
Jandhyala SM, Talukdar R, Subramanyam C, Vuyyuru H, Sasikala M, and Reddy DN. 2015. Role of the normal gut microbiota. World journal of gastroenterology: WJG 21:8787.

Jobling M. 2012. National Research Council (NRC): Nutrient requirements of fish and shrimp. Springer.

Katoh K, and Standley DM. 2013. MAFFT multiple sequence alignment software version 7: improvements in performance and usability. Molecular biology and evolution 30:772-780.

Li J, Jiang H, Li L, Zhang X, and Chen J. 2019. The effect of disease and season to hepatopancreas and intestinal mycobiota of Litopenaeus vannamei. Frontiers in Microbiology 10:889-889. https://doi.org/10.3389/fmicb.2019.00889

Lin H, and Peddada SD. 2020. Analysis of compositions of microbiomes with bias correction. Nature Communications 11:3514. https://doi.org/10.1038/s41467-020-17041-7

Liu J, Wang K, Wang Y, Chen W, Jin Z, Yao Z, and Zhang D. 2019. Strain-specific changes in the gut microbiota profiles of the white shrimp Litopenaeus vannamei in response to cold stress. Aquaculture 503:357-366. https://doi.org/10.1016/i.aquaculture.2019.01.026

Lu X, Kong J, Luan S, Dai P, Meng X, Cao B, and Luo K. 2016. Transcriptome analysis of the hepatopancreas in the pacific white shrimp (Litopenaeus vannamei) under acute ammonia stress. PloS one 11:e0164396. https://doi.org/10.1371/journal.pone.0164396

Ma S, Kim A, Lee W, Kim S, Lee S, Yoon D, Bae J-S, Park C-I, and Kim S. 2020. Vibrio harveyi infection significantly alters amino acid and carbohydrate metabolism in whiteleg shrimp, Litopenaeus vannamei. Metabolites 10:265. https://doi.org/10.3390/metabo10060265

Marín I, and Arahal DR. 2014. The Family Beijerinckiaceae. In: Rosenberg E, DeLong EF, Lory S, Stackebrandt E, and Thompson F, eds. The Prokaryotes: Alphaproteobacteria and Betaproteobacteria. Berlin, Heidelberg: Springer Berlin Heidelberg, 115-133.

McDonald R, Zhang F, Watts JEM, and Schreier HJ. 2015. Nitrogenase diversity and activity in the gastrointestinal tract of the wood-eating catfish Panaque nigrolineatus. The ISME Journal 9:2712-2724. https://doi.org/10.1038/ismej.2015.65

McGaw IJ, and Curtis DL. 2013. A review of gastric processing in decapod crustaceans. Journal of Comparative Physiology B 183:443-465.

Muhammad F, Zhang ZF, Shao MY, Dong YP, and Muhammad S. 2012. Ontogenesis of digestive system in Litopenaeus vannamei (Boone, 1931) (Crustacea: Decapoda). Italian Journal of Zoology 79:7785. https://doi.org/10.1080/11250003.2011.590534

Nielsen S, Walburn JW, Verges A, Thomas T, and Egan S. 2017. Microbiome patterns across the gastrointestinal tract of the rabbitfish Siganus fuscescens. PeerJ 5:e3317.

Ortiz-Estrada ÁM, Martínez-Porchas M, Martínez-Córdova LR, Vargas-Albores F, Burgos-Hernandéz A, Scheuren-Acevedo SM, and Gollas-Galván T. 2021. Bacterial communities and predicted nitrogen metabolism of heterotrophic- and probiotic-based biofilms used for super-intensive indoor shrimp culture. Aquaculture Research 52:334-344. https://doi.org/10.1111/are.14896

Palacios E, Bonilla A, Luna D, and Racotta IS. 2004. Survival, Na+/K+-ATPase and lipid responses to salinity challenge in fed and starved white pacific shrimp (Litopenaeus vannamei) postlarvae. Aquaculture 234:497-511.

Price MN, Dehal PS, and Arkin AP. 2010. FastTree 2-approximately maximum-likelihood trees for large alignments. PloS one 5:e9490.

Pujalte MJ, Lucena T, Ruvira MA, Arahal DR, and Macián MC. 2014. The Family Rhodobacteraceae. The Prokaryotes. Berlin, Heidelberg: Springer, 439-512.

Robles-Porchas GR, Gollas-Galván T, Martínez-Porchas M, Martínez-Cordova LR, Miranda-Baeza A, and Vargas-Albores F. 2020. The nitrification process for nitrogen removal in biofloc system aquaculture. Reviews in Aquaculture 12:2228-2249. https://doi.org/10.1111/raq.12431

Rosas C, Cuzon G, Gaxiola G, Arena L, Lemaire P, Soyez C, and Van Wormhoudt A. 2000. Influence of dietary carbohydrate on the metabolism of juvenile Litopenaeus stylirostris. Journal of

Peer] reviewing PDF | (2021:03:59686:2:0:NEW 23 Jun 2021) 
687

688

689

690

691

692

693

694

695

696

697

698

699

700

701

702

703

704

705

706

707

708

709

710

711

712

713

714

715

716

717

718

719

720

721

722

723

724

725

726

727

728

729

730

731

732

Experimental Marine Biology and Ecology 249:181-198. https://doi.org/10.1016/S00220981(00)00184-2

Rőszer T. 2014. The invertebrate midintestinal gland ("hepatopancreas") is an evolutionary forerunner in the integration of immunity and metabolism. Cell Tissue Res 358:685-695.

https://doi.org/10.1007/s00441-014-1985-7

Rowland I, Gibson G, Heinken A, Scott K, Swann J, Thiele I, and Tuohy K. 2018. Gut microbiota functions: metabolism of nutrients and other food components. European journal of nutrition 57:1-24. https://doi.org/10.1007/s00394-017-1445-8

Sañudo-Wilhelmy SA, Gómez-Consarnau L, Suffridge C, and Webb EA. 2014. The role of B vitamins in marine biogeochemistry. Annual Review of Marine Science 6:339-367. https://doi.org/10.1146/annurev-marine-120710-100912

Schrader C, Schielke A, Ellerbroek L, and Johne R. 2012. PCR inhibitors - occurrence, properties and removal. Journal of Applied Microbiology 113:1014-1026. https://doi.org/10.1111/j.13652672.2012.05384.x

Sommer F, and Bäckhed F. 2013. The gut microbiota - masters of host development and physiology. Nature Reviews Microbiology 11:227. https://doi.org/10.1038/nrmicro2974

Soonthornchai W, Chaiyapechara S, Jarayabhand P, Söderhäll K, and Jiravanichpaisal P. 2015. Interaction of Vibrio spp. with the inner surface of the digestive tract of Penaeus monodon. PloS one 10:e0135783. https://doi.org/10.1371/journal.pone.0135783

Stackebrandt E, and Schumann P. 2014. The Family Cellulomonadaceae. In: Rosenberg E, DeLong EF, Lory S, Stackebrandt E, and Thompson F, eds. The Prokaryotes: Actinobacteria. Berlin, Heidelberg: Springer Berlin Heidelberg, 163-184.

Su F, Huang B, and Liu J. 2018. The carotenoids of shrimps (Decapoda: Caridea and Dendrobranchiata) cultured in China. Journal of Crustacean Biology 38:523-530. https://doi.org/10.1093/jcbiol/ruy049

Tanumihardjo SA. 2012. Carotenoids and human health: Springer Science \& Business Media.

Tzuc JT, Escalante DR, Rojas Herrera R, Gaxiola Cortés G, and Ortiz MLA. 2014. Microbiota from Litopenaeus vannamei: digestive tract microbial community of Pacific white shrimp (Litopenaeus vannamei). SpringerPlus 3:280-280. https://doi.org/10.1186/2193-1801-3-280

Wang J, Zhao W, Liang Y, Han Y, Wang X, Zhang X, Ma X, Zhang D, and Huang Z. 2017a. Development of a prototype $16 \mathrm{~S}$ rRNA gene-based microarray for monitoring planktonic actinobacteria in shrimp ponds. International Aquatic Research 9:71-80.

Wang X, Li E, Xu Z, Li T, Xu C, and Chen L. 2017b. Molecular response of carbohydrate metabolism to dietary carbohydrate and acute low salinity stress in Pacific white shrimp Litopenaeus vannamei. Turkish Journal of Fisheries and Aquatic Sciences 17:153-169.

Wu Z, Zhang Q, Zhang T, Chen J, Wang S, Hao J, Lin Y, and Li A. 2021. Association of the microbiota dysbiosis in the hepatopancreas of farmed crayfish (Procambarus clarkii) with disease outbreaks. Aquaculture 536:736492. https://doi.org//10.1016/j.aquaculture.2021.736492

Zheng Y, Yu M, Liu J, Qiao Y, Wang L, Li Z, Zhang X-H, and Yu M. 2017. Bacterial community associated with healthy and diseased pacific white shrimp (Litopenaeus vannamei) larvae and rearing water across different growth stages. Frontiers in Microbiology 8:1362. https://doi.org/10.3389/fmicb.2017.01362

Zimmer M, Danko JP, Pennings SC, Danford AR, Ziegler A, Uglow RF, and Carefoot TH. 2001. Hepatopancreatic endosymbionts in coastal isopods (Crustacea: Isopoda), and their contribution to digestion. Marine Biology 138:955-963. https://doi.org/10.1007/s002270000519 
734 Table 1. Enriched KEGG pathways (level 1, 2, and 3) according to the digestive tract microbiota

735 of the digestive tract of white shrimp (Litopenaeus vannamei) with significant differences $(\mathrm{p}<0.05)$

736 based on the Kruskal-Wallis test.

737

738 
739 Figure 1. Alfa diversity of the microbiota from the three digestive tract fractions of the white

740 shrimp (Litopenaeus vannamei). The Chao, Observed and Shannon indexes were calculated based

741 on different ASVs resolution levels. A) Indexes were calculated based on the Raw ASVs count.

742 B) Indexes calculated based on the ASVs clustered at the family level.

743 Figure 2. The occurrence of families across the digestive tract fractions of the white shrimp

744 (Litopenaeus vannamei). The numbers at the top of the bars are the size of intersections. The

745 connected dots in the bottom of the bars correspond to the families intersected through the three

746 fractions. The non-connected dots (colored) are the exclusive families in one of the fractions.

747 Figure 3. Relative abundance of microbiota enriched in the digestive tract fractions of the white

748 shrimp (Litopenaeus vannamei) at the phylum level. Abundance $<1 \%$ was grouped in the low 749 abundance category.

750

751 Figure 4. Relative abundance bar plot with the differential abundance (DA) (significance $<0.05$ )

752 microbial families enriched in the digestive tract fractions of the white shrimp (Litopenaeus 753 vannamei). Y-axis is ordered as in figure 6.

Figure 5. Principal coordinate analysis (PCoA) plot based on weighted UniFrac (A), Jaccard (B), and Bray Curtis distance measurements of beta diversity associated with the gut microbiota of the white shrimp (Litopenaeus vannamei). The Foregut (stomach) is represented by red color; blue corresponds to the Midgut (hepatopancreas), and yellow belongs to the Hindgut (intestine). 
760 Figure 6. Differential abundance (DA) analysis with bias correction of the significant families

761 among the digestive tract fractions. * significant at 5\% level of significance; $* *$ significant at $1 \%$

762 level of significance; ***significant at $0.1 \%$ level of significance.

Figure 7. Network interaction graph for microbial communities of the Foregut fraction at the order level, using weighted topological (wTO) network analysis. Blue color links are positive correlations, while negative correlations are in red color links. The shade of the links means how strong the interaction is, as well as the degree of interaction within the orders is represented by the node size.

Figure 8. Network interaction graph for microbial communities of the Midgut fraction at the order level, using weighted topological (wTO) network analysis. Blue color links are positive correlations, while negative correlations are in coral color links. The shade of the links means how strong the interaction is, as well as the degree of interaction within the orders is represented by the node size.

Figure 9. Network interaction graph for microbial communities of the Hindgut at the order level, using weighted topological (wTO) network analysis. Blue color links are positive correlations, while negative correlations are in red color links. The shade of the links means how strong the interaction is, as well as the degree of interaction within the orders is represented by the node size.

Figure 10. Heatmap of microbial functions from fractions of the digestive tract from white shrimp 
783 Wallis H test was used to analyze the significant differences of species among four groups. KEGG,

784 Kyoto Encyclopedia of Genes and Genomes. Values from the color scale represented de 785 abundance of predicted functions annotated for each KEGG level-1 category. 
Figure 1

Alpha diversity of the microbiota from the three digestive tract fractions of the white shrimp (Litopenaeus vannamei)

The Chao, Observed, and Shannon indexes were calculated based on different ASVs resolution levels. A) Indexes were calculated based on the Raw ASVs count. B). Indexes calculated based on the ASVs clustered at the family level. 

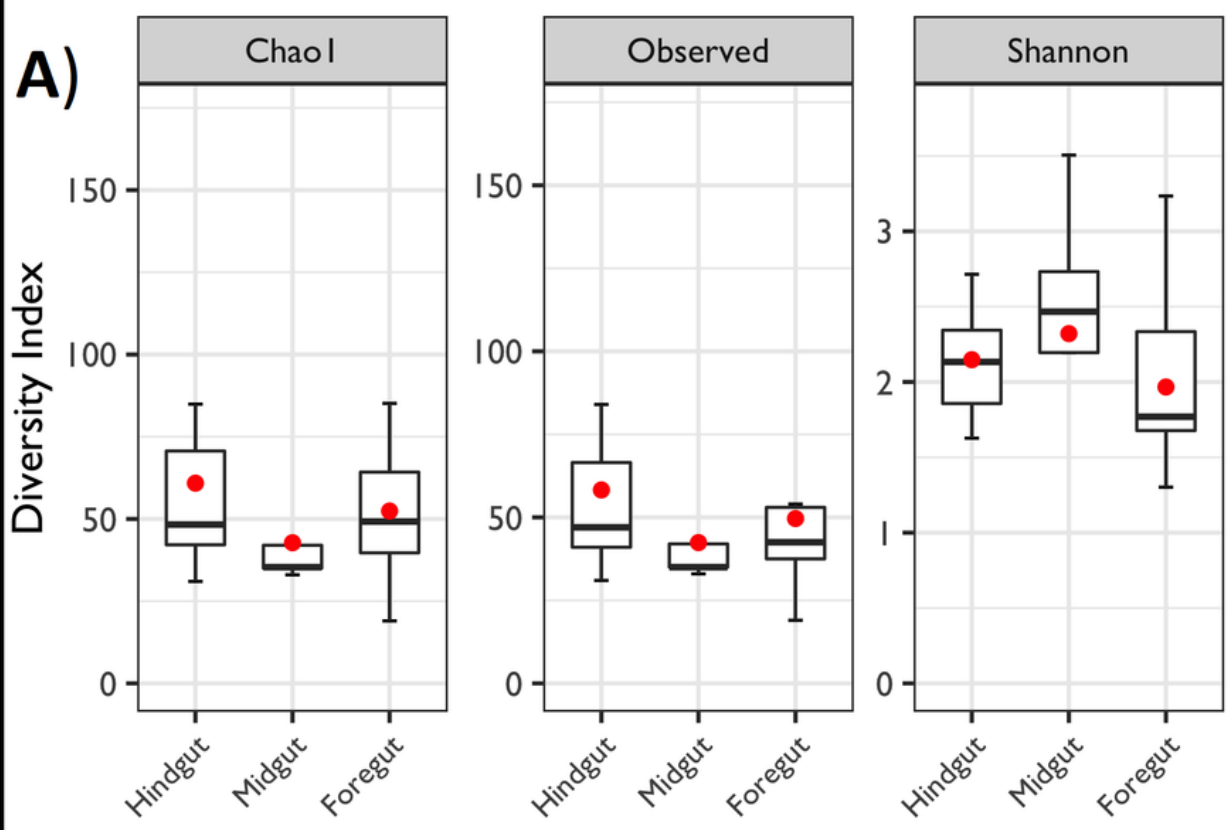

Red dots $=$ mean
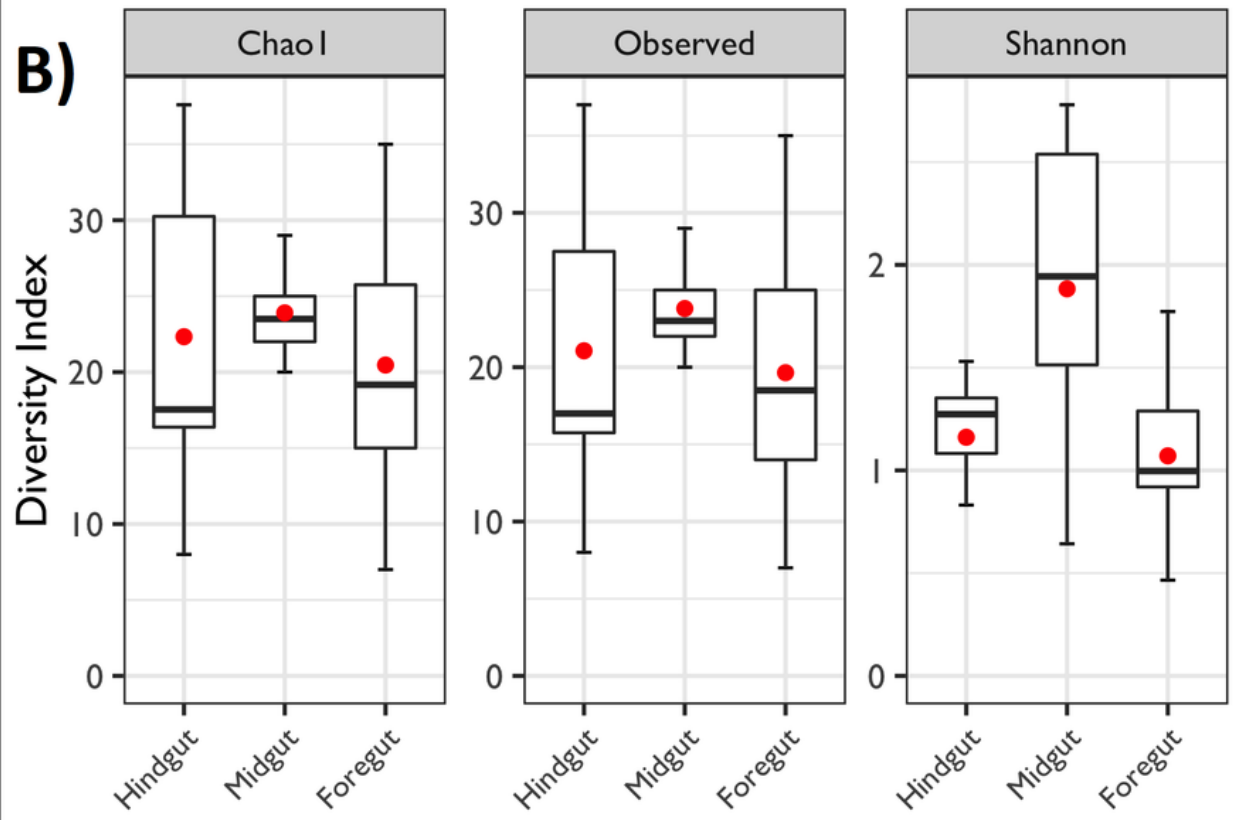

Red dots $=$ mean

Anova, $\mathrm{F}(2,32)=5.7, \mathrm{p}=0.008, \eta_{g}^{2}=0.026$ 


\section{Figure 2}

The occurrence of families across the digestive tract fractions of the white shrimp (Litopenaeus vannamei).

The numbers at the top of the bars are the size of intersections. The connected dots in the bottom of the bars correspond to the families intersected through the three fractions. The non-connected dots (colored) are the exclusive families in one of the fractions. 


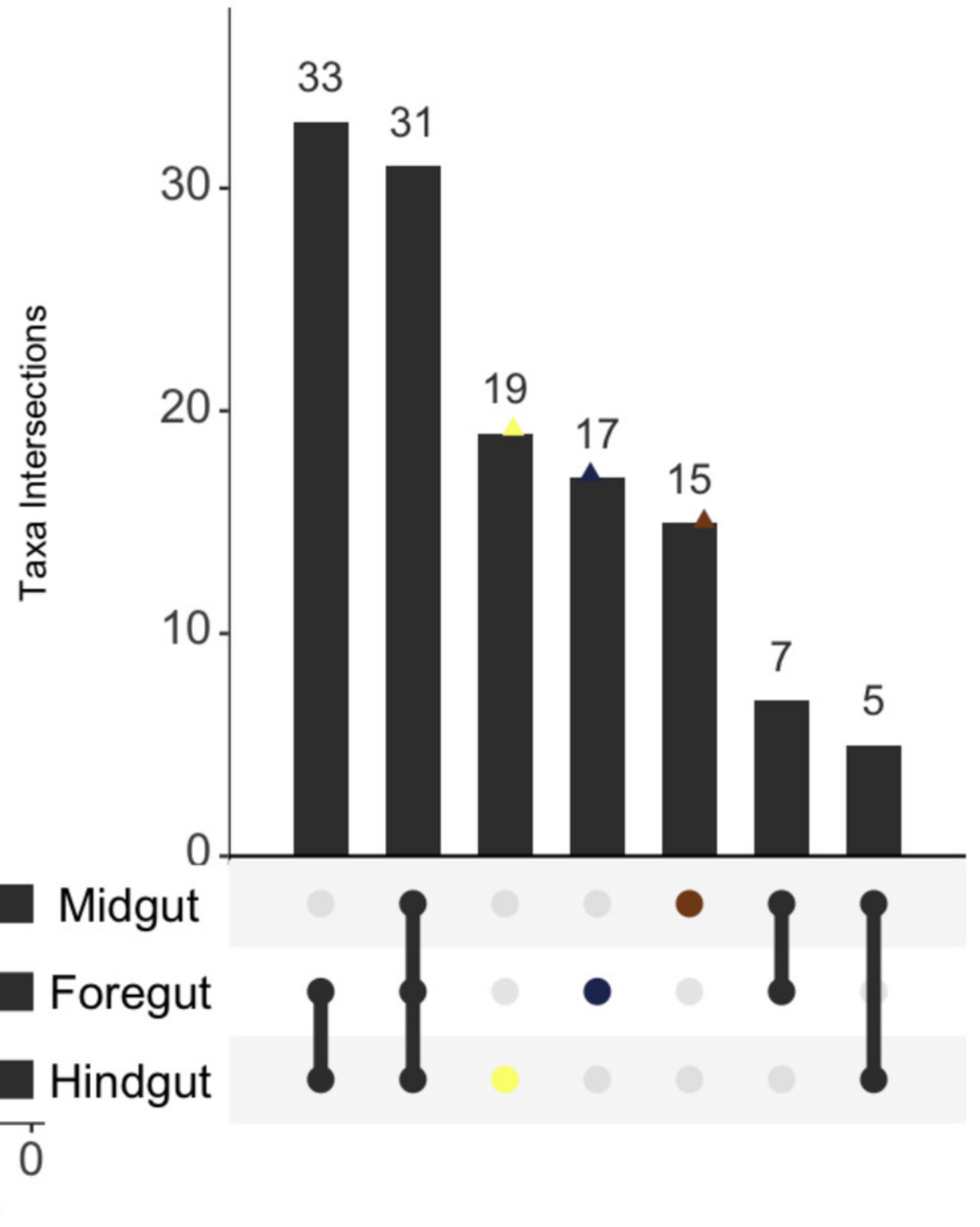




\section{Figure 3}

Relative abundance of microbiota enriched in the digestive tract fractions of the white shrimp (Litopenaeus vannamei) at the phylum level.

Abundance $<1 \%$ was grouped in the low abundance category.

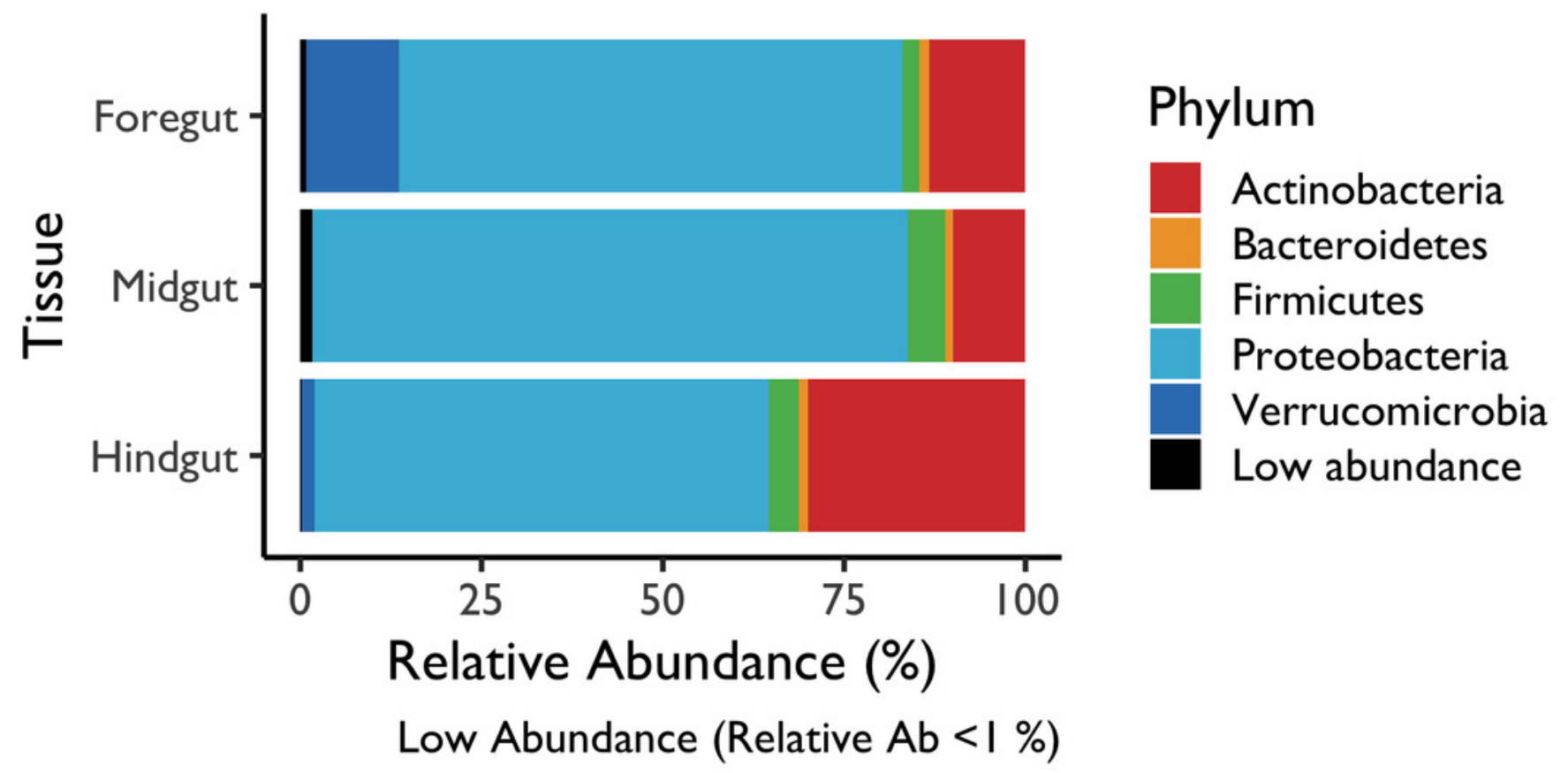


Figure 4

Relative abundance bar plot with the differential abundance (DA) (significance $<0.05$ ) microbial families enriched in the digestive tract fractions of the White shrimp (Litopenaeus vannamei).

Y-axis is ordered as in figure 5. 


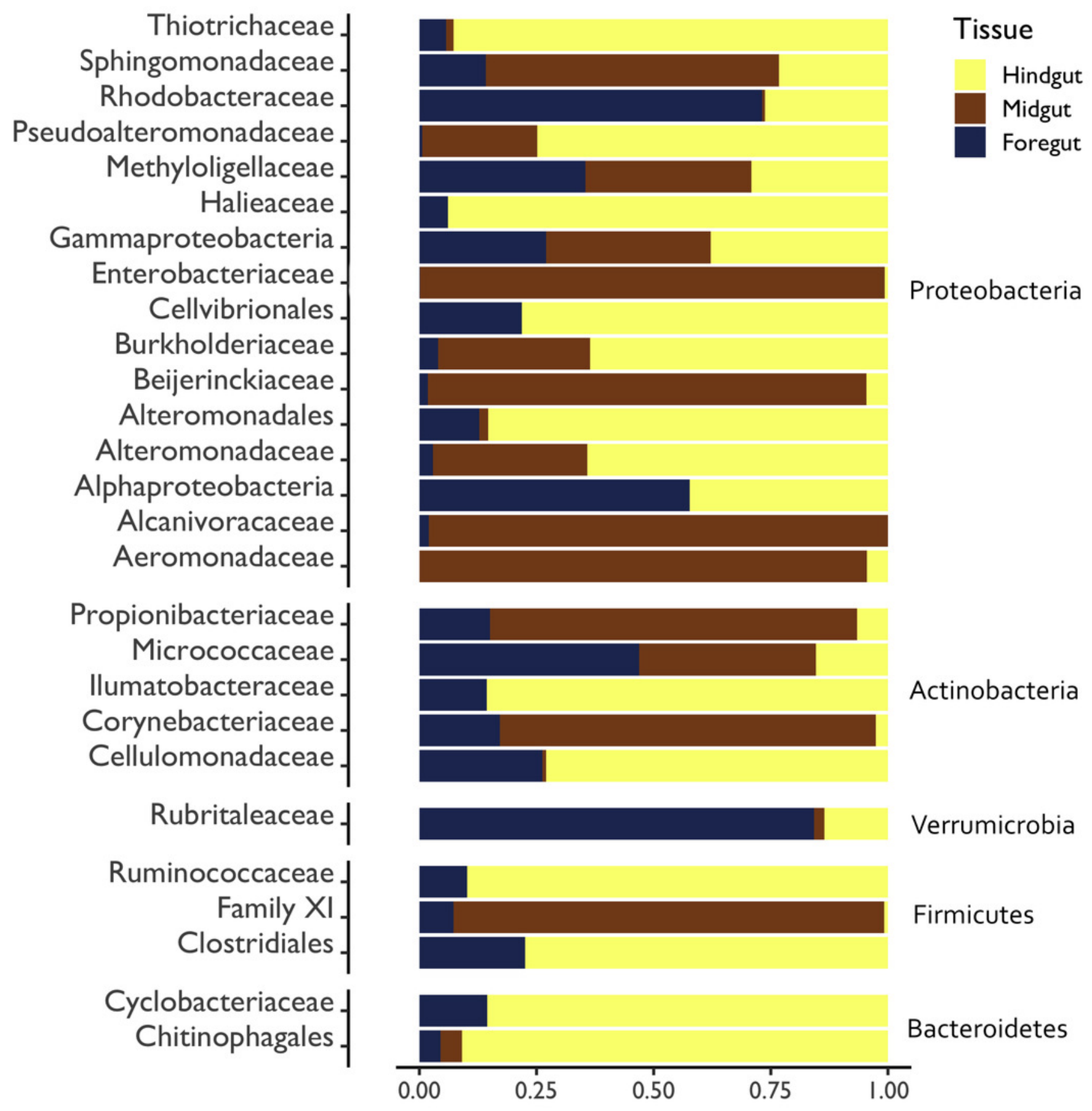


Figure 5

Principal coordinate analysis (PCoA) plot based on weighted UniFrac (A), Jaccard (B), and Bray Curtis distance measurements of beta diversity associated with the gut microbiota of the White shrimp (Litopenaeus vannamei).

The Foregut (stomach) is represented by red color; blue corresponds to the Midgut (hepatopancreas), and yellow belongs to the Hindgut (intestine).
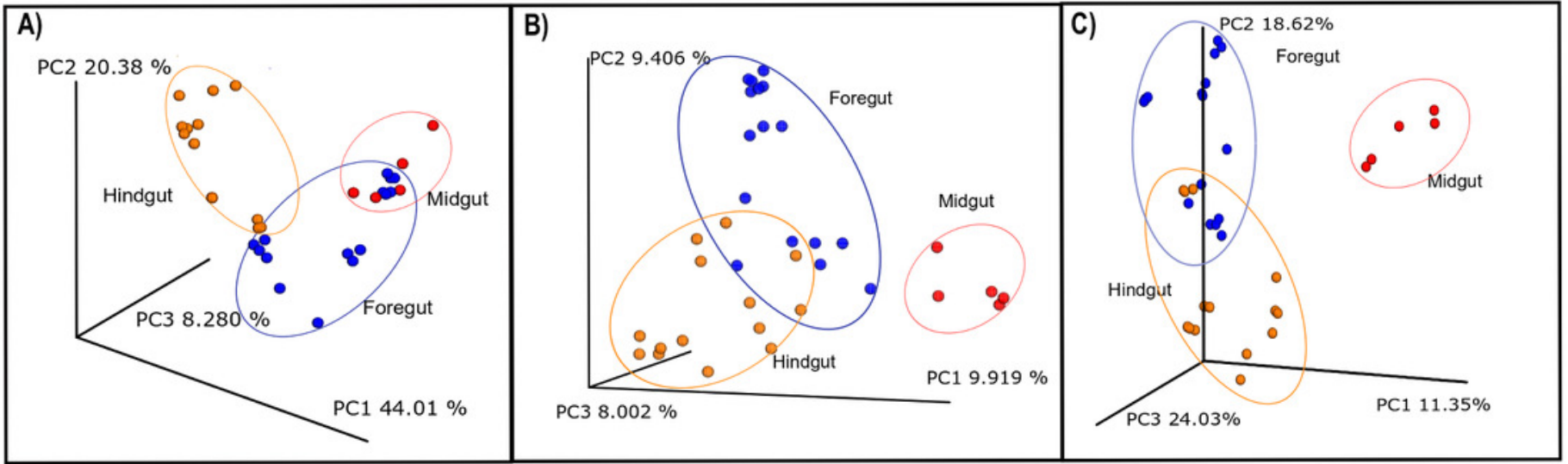
Figure 6

Differential abundance (DA) analysis with bias correction of the significant families among the digestive tract fractions.

* significant at $5 \%$ level of significance; ${ }^{* *}$ significant at $1 \%$ level of significance;

$* * *$ significant at $0.1 \%$ level of significance. 


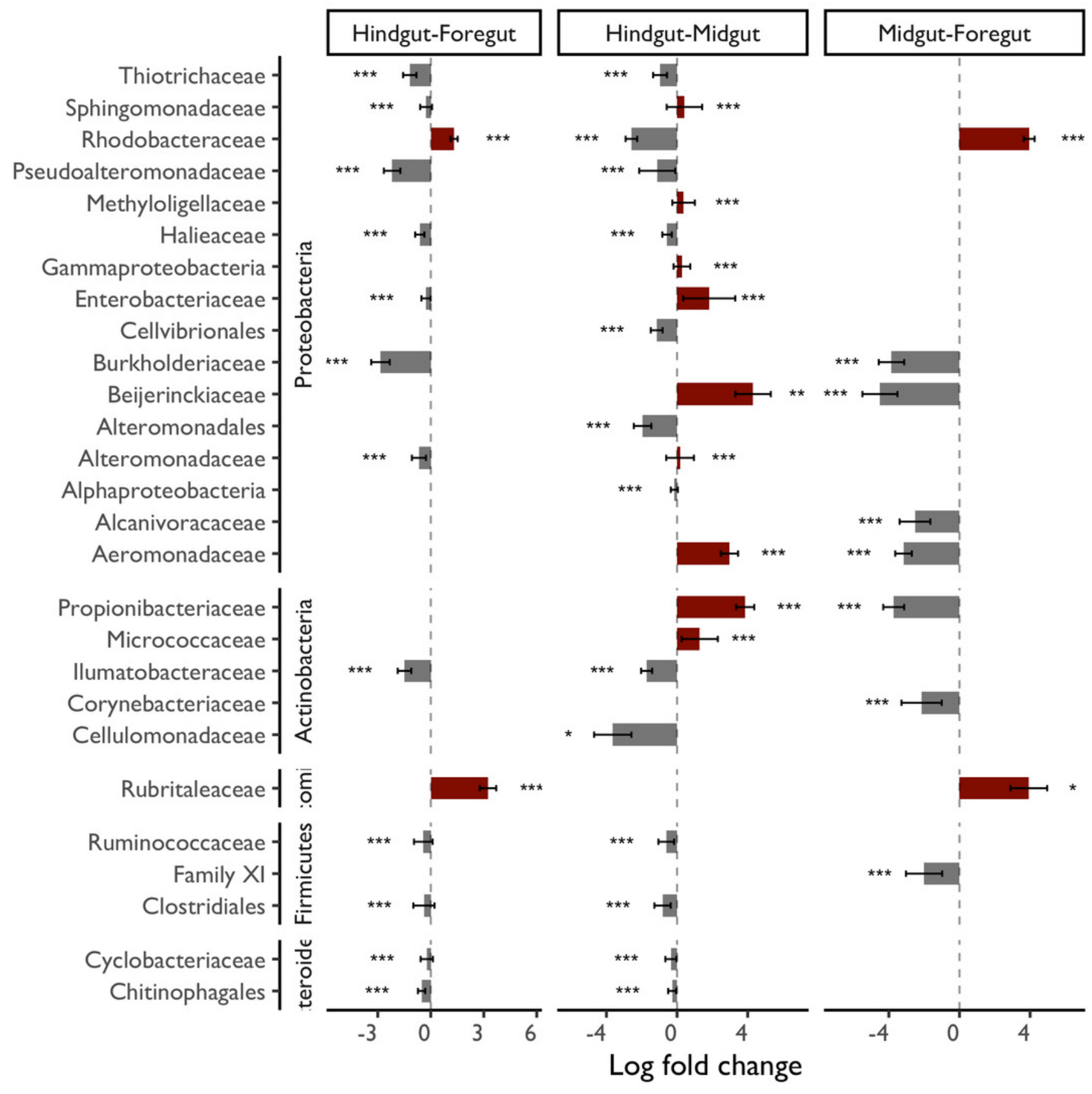




\section{Figure 7}

Network interaction graph for microbial communities of the Midgut fraction at the order level, using weighted topological (wTO) network analysis.

Blue color links are positive correlations, while negative correlations are in coral color links. The shade of the links means how strong the interaction is, as well as the degree of interaction within the orders is represented by the node size.

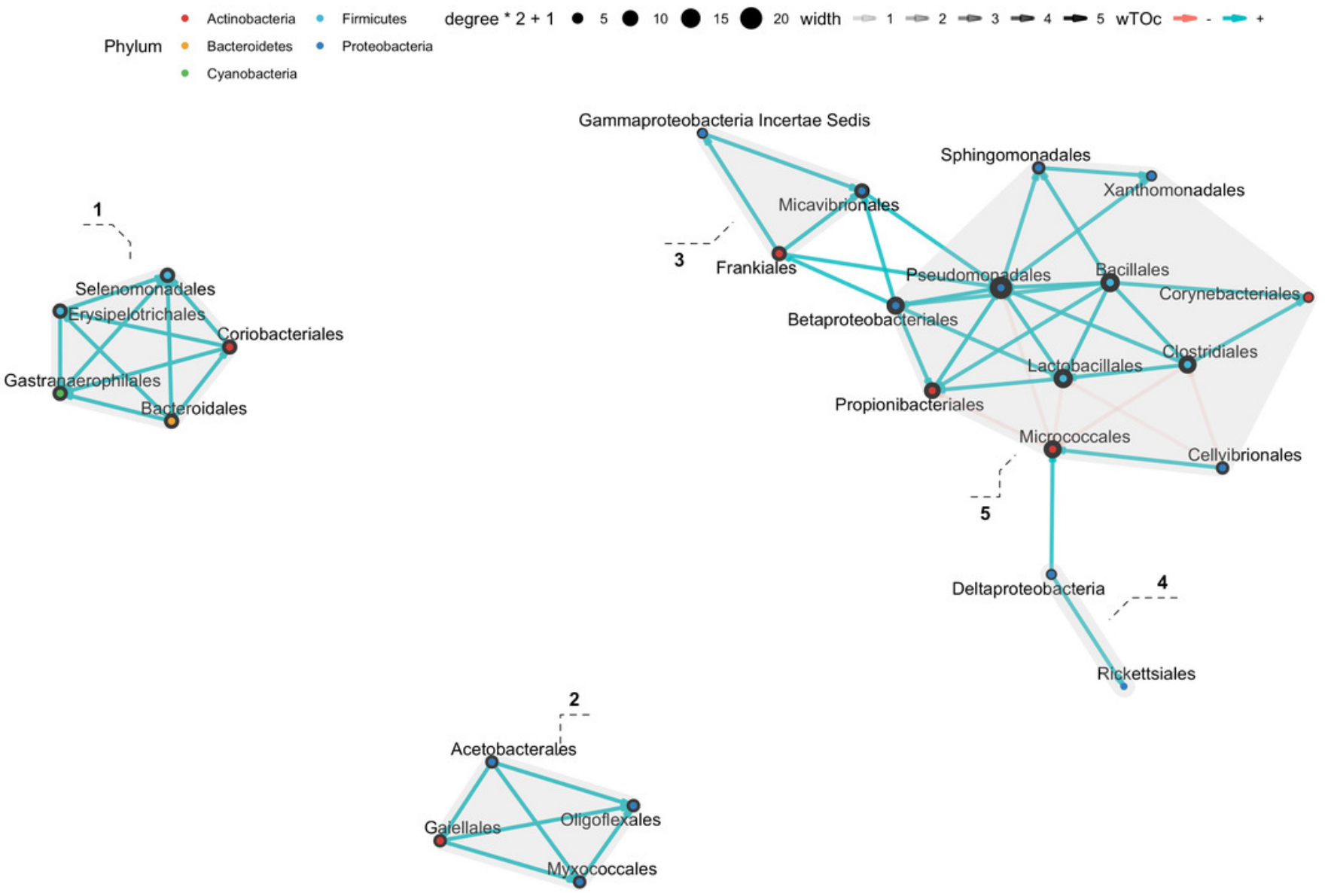


Figure 8

Network interaction graph for microbial communities of the Hindgut at the order level, using weighted topological (wTO) network analysis.

Blue color links are positive correlations, while negative correlations are in red color links. The shade of the links means how strong the interaction is, as well as the degree of interaction within the orders is represented by the node size.

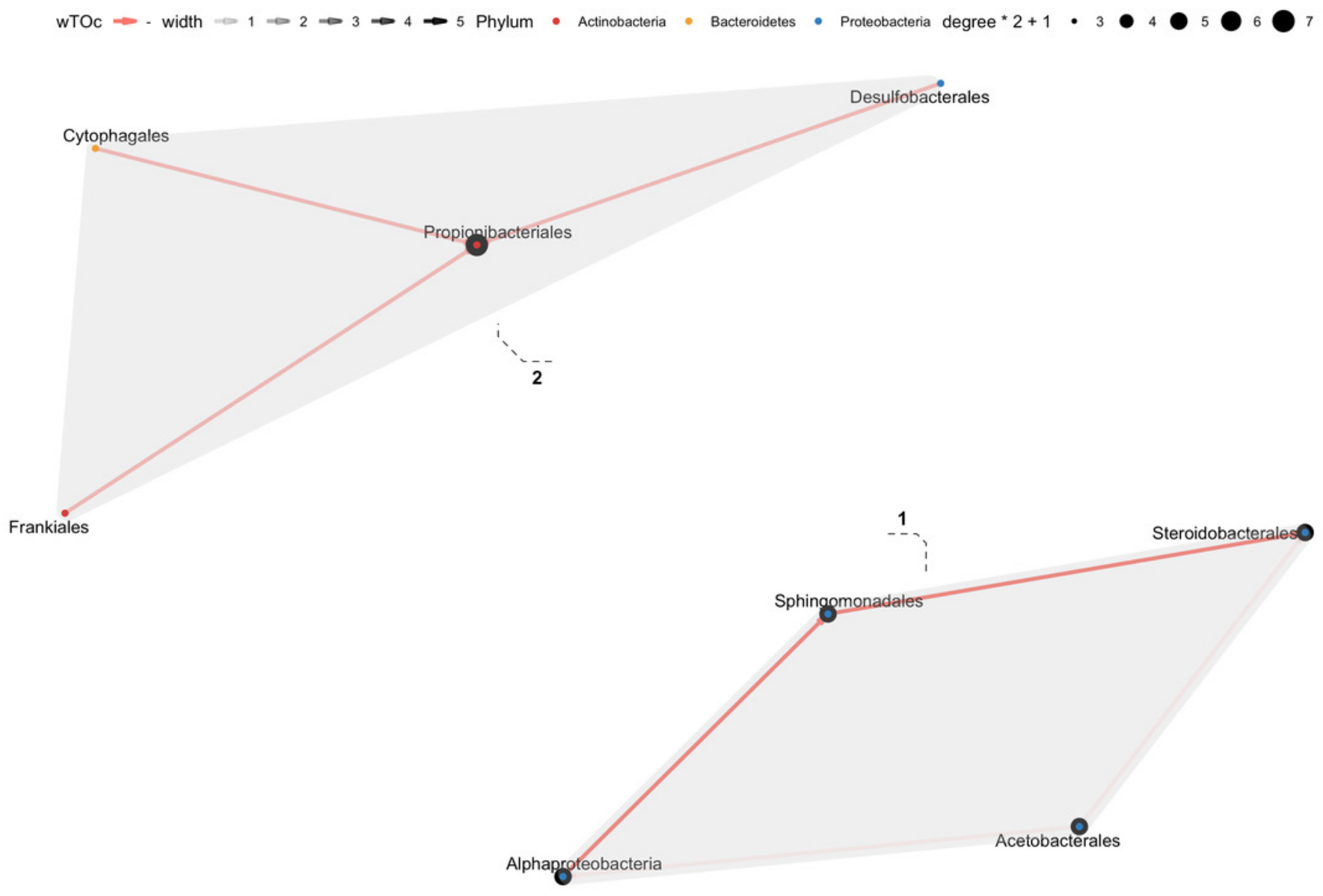


Figure 9

Network interaction graph for the hindgut microbial communities at the order level, using weighted topological (wTO) network analysis.

Blue color links are positive correlations, while negative correlations are in red color links. The shade of the links means how strong the interaction is, as well as the degree of interaction within the orders is represented by the node size.

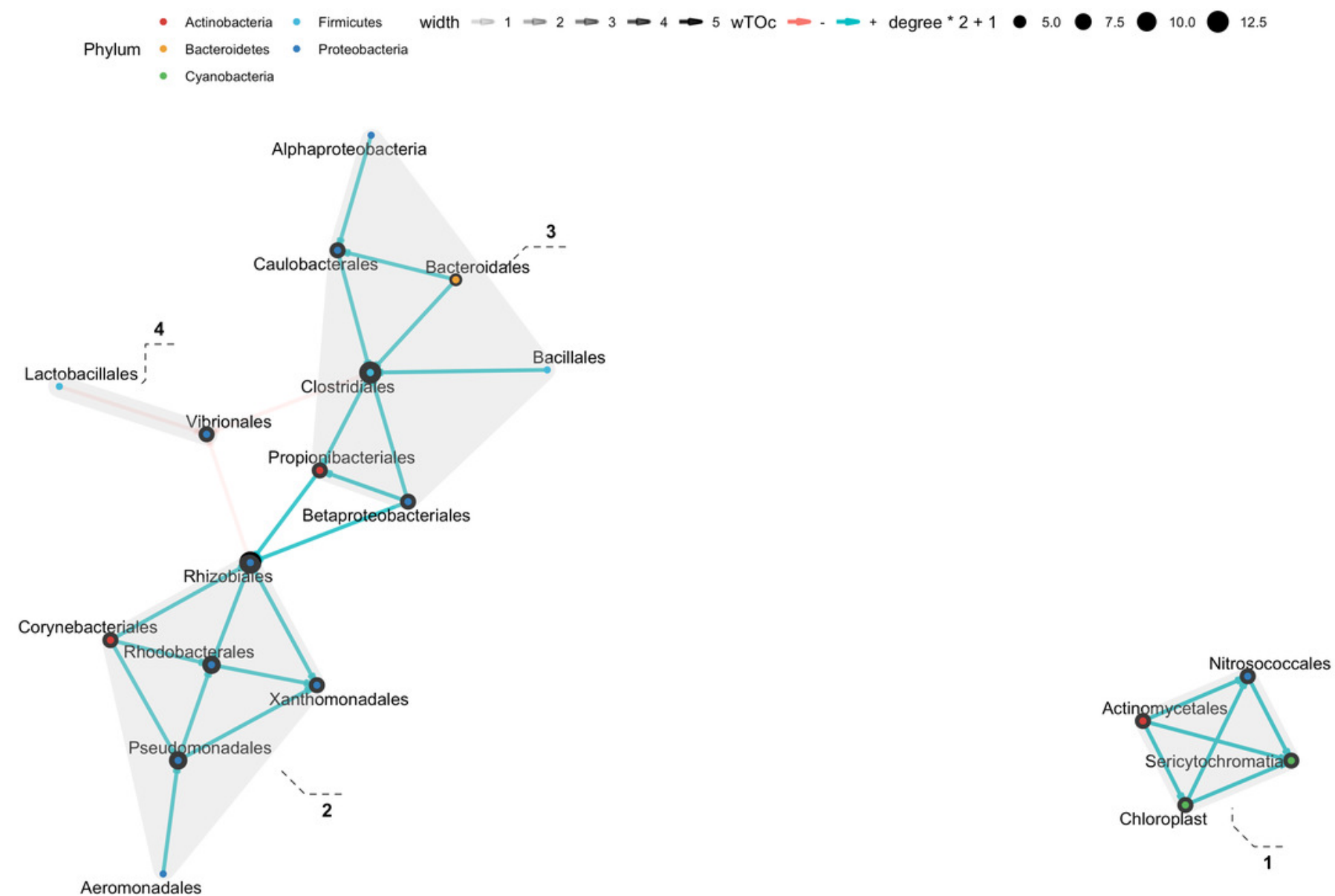




\section{Figure 10}

Heatmap of microbial functions from fractions of the digestive tract of the white shrimp (Litopenaeus vannamei), predicted using level-1 and level 2 of the KEGG database.

Kruskal-Wallis $\mathrm{H}$ test was used to analyze the significant differences of species among four groups. KEGG, Kyoto Encyclopedia of Genes and Genomes. Values from the color scale represented the abundance of predicted functions annotated for each KEGG level-1 category.

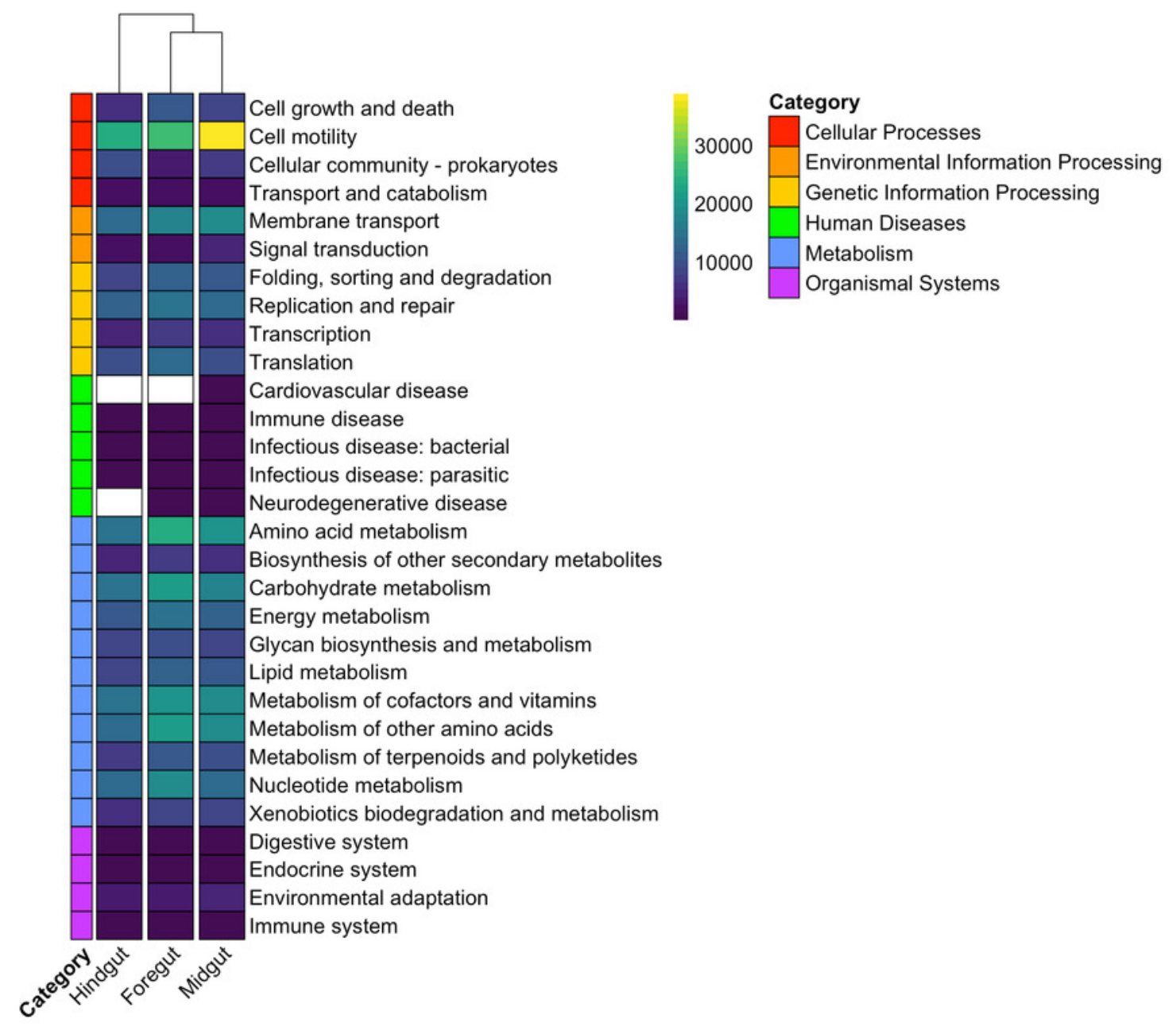




\section{Table $\mathbf{1}$ (on next page)}

Enriched KEGG pathways (level 1, 2, and 3) according to the digestive tract microbiota of the digestive tract of white shrimp (Litopenaeus vannamei) with significant differences $(p<0.05)$ based on the Kruskal-Wallis test. 
1 Table 1. Enriched KEGG pathways (level 1, 2, and 3) according to the digestive tract microbiota

2 of the digestive tract of white shrimp (Litopenaeus vannamei) with significant differences $(\mathrm{p}<0.05)$

3 based on the Kruskal-Wallis test.

\begin{tabular}{|c|c|c|}
\hline Fraction & KEGG Level-1 and Level-2 & KEGG Level-3 \\
\hline Foregut & $\begin{array}{l}\text { Metabolism; Amino acid metabolism } \\
\text { Metabolism; Amino acid metabolism } \\
\text { Metabolism; Amino acid metabolism } \\
\text { Metabolism; Amino acid metabolism } \\
\text { Metabolism; Carbohydrate metabolism } \\
\text { Metabolism; Carbohydrate metabolism } \\
\text { Metabolism; Energy metabolism } \\
\text { Metabolism; Metabolism of other amino acids } \\
\text { Metabolism; Xenobiotics biodegradation and } \\
\text { metabolism } \\
\text { Metabolism; Xenobiotics biodegradation and } \\
\text { metabolism } \\
\text { Cellular Processes; Cell growth and death } \\
\text { Ribosome biogenesis in eukaryotes; Translation }\end{array}$ & $\begin{array}{l}\text { Glycine, serine and threonine metabolism } \\
\text { Valine, leucine and isoleucine biosynthesis } \\
\text { Arginine and proline metabolism } \\
\text { Lysine degradation } \\
\text { C5-Branched dibasic acid metabolism } \\
\text { Glyoxylate and dicarboxylate metabolism } \\
\text { Carbon fixation pathways in prokaryotes } \\
\text { D-Arginine and D-ornithine metabolism } \\
\text { Chloroalkane and chloroalkene degradation } \\
\text { Polycyclic aromatic hydrocarbon degradation } \\
\text { Apostosis } \\
\text { Ribosome biogenesis in eukaryotes }\end{array}$ \\
\hline Midgut & $\begin{array}{l}\text { Genetic Information Processing; Folding, sorting } \\
\text { and degradation } \\
\text { Genetic Information Processing; Folding, sorting } \\
\text { and degradation } \\
\text { Metabolism; Amino acid metabolism } \\
\text { Metabolism; Lipid metabolism } \\
\text { Metabolism; Metabolism of cofactors and } \\
\text { vitamins } \\
\text { Metabolism; Metabolism of cofactors and } \\
\text { vitamins } \\
\text { Metabolism; Metabolism of terpenoids and } \\
\text { polyketides } \\
\text { Organismal Systems; Endocrime system } \\
\text { Metabolism; Xenobiotics biodegradation and } \\
\text { metabolism }\end{array}$ & $\begin{array}{l}\text { Protein processing in endoplasmic reticulum } \\
\text { Sulfur relay system } \\
\text { Tryptophan metabolism } \\
\text { Glycerophospholipid metabolism } \\
\text { Folate biosynthesis } \\
\text { Vitamin B6 metabolism } \\
\text { Carotenoid biosynthesis } \\
\text { Insulin signaling pathway } \\
\text { Toluene degradation }\end{array}$ \\
\hline Hindgut & $\begin{array}{l}\text { Metabolism; Metabolism of cofactors and } \\
\text { vitamins } \\
\text { Environmental Information Processing; } \\
\text { Membrane transport } \\
\text { Metabolism; Energy metabolism } \\
\text { Metabolism; Metabolism of cofactors and } \\
\text { vitamins } \\
\text { Metabolism; Xenobiotics biodegradation and } \\
\text { metabolism }\end{array}$ & $\begin{array}{l}\text { Nicotinate and nicotinamide metabolism } \\
\text { Phosphotransferase system (PTS) } \\
\text { Nitrogen metabolism } \\
\text { Ubiquinone and other terpenoid-quinone biosynthesis } \\
\text { Drug metabolism - other enzyme }\end{array}$ \\
\hline
\end{tabular}


Genetic Information Processing; Replication and repair 